\title{
Abnormal CD8 T cells induce and track Alzheimer's- like neurodegeneration
}

Christopher Wheeler ( $\nabla$ chris.wheeler@brainmappingfoundation.org )

T-Neuro Pharma https://orcid.org/0000-0003-0618-8748

\section{Akanksha Panwar}

Cedars-Sinai Medical Center

Altan Rentsendorj

Cedars-Sinai Medical Center

Michelle Jhun

Cedars-Sinai Medical Center

Robert Cohen

Emory University School of Medicine

Ryan Cordner

Brigham Young Univesity

Nicole Gull

Cedars-Sinai Medical Center https://orcid.org/0000-0002-1956-5648

Robert Pechnick

Western University of Health Sciences

Gretchen Duvall

Cedars-Sinai Medical Center

Armen Mardiros

Cedars-Sinai Medical Center

David Golchian

Cedars-Sinai Medical Center

Hannah Schubloom

Cedars-Sinai Medical Center

Lee-way Jin

University of California, Davis

Debby Van Dam

University of Antwerp

Yannick Vermeiren

Institue Born-Bunge, University of Antwerp

Hans De Reu

University of Antwerp

Peter Paul De Deyn 
Institue Born-Bunge, University of Antwerp

Keith Black

Cedars-Sinai Medical Center

\section{Biological Sciences - Article}

Keywords: Alzheimer's disease, neurodegeneration, CD8 T cells

Posted Date: February 26th, 2021

DOl: https://doi.org/10.21203/rs.3.rs-199647/v1

License: (c) (i) This work is licensed under a Creative Commons Attribution 4.0 International License. Read Full License 


\section{Abnormal CD8 T cells induce and track Alzheimer's-like neurodegeneration}

2 Akanksha Panwar ${ }^{\mathrm{a},{ }^{*}}$, Altan Rentsendorj ${ }^{\mathrm{a},{ }^{,},}$, Michelle Jhun ${ }^{\mathrm{a},{ }^{*},}$, Robert M. Cohen ${ }^{\mathrm{b}}$, Ryan Cordner ${ }^{\mathrm{a}, \mathrm{c}, \dagger}$,

3 Nicole Gull ${ }^{\mathrm{c}}$, Robert N. Pechnick ${ }^{\mathrm{d}}$, Gretchen Duvall ${ }^{\mathrm{a}}$, Armen Mardiros ${ }^{\mathrm{a}, \uparrow}{ }^{\dagger}$, David Golchian ${ }^{\mathrm{a}}$, Hanna

4 Schubloom $^{\mathrm{a}}$, Lee-Way Jin ${ }^{\mathrm{e}}$, Debby Van Dam ${ }^{\mathrm{f}}$, Yannick Vermeiren ${ }^{\mathrm{f}}$, Hans De Reu ${ }^{\mathrm{g}}$, Peter Paul De

$5 \quad$ Deyn $^{\mathrm{h}}$, Keith L. Black ${ }^{\mathrm{a}}$, and Christopher J. Wheeler ${ }^{\mathrm{a}, \mathrm{i}}$

$6 \quad{ }^{a}$ Dept. Neurosurgery, Maxine Dunitz Neurosurgical Institute, Cedars-Sinai Medical Center, 8700

7 Beverly Blvd., Los Angeles, CA 90048.

$8 \quad{ }^{b}$ Dept. Psychiatry \& Behavioral Sciences and Neuroscience Program, GDBBS, Emory University,

9201 Dowman Dr., Atlanta, GA 30322.

$10 \quad$ 'Dept. Biomedical \& Translational Sciences, Cedars-Sinai Medical Center, 8700 Beverly Blvd., 11 Los Angeles, CA 90048.

$12 \quad{ }^{\mathrm{d}}$ Dept. of Basic Medical Sciences, College of Osteopathic Medicine of the Pacific, Western

13 University of Health Sciences, 309 E. $2^{\text {nd }}$ St., Pomona, CA 91766.

$14{ }^{\mathrm{e}}$ Dept. Medical Pathology and Laboratory Medicine, M.I.N.D. Institute, University of California, 15 Davis, $282550^{\text {th }}$ Street, Sacramento, CA 95817.

16 fLaboratory of Neurochemistry and Behavior, Department of Biomedical Sciences, Institute Born-

17 Bunge, University of Antwerp, Antwerp, Belgium; and

18 Department of Neurology and Alzheimer Research Center, University of Groningen and

19 University Medical Center Groningen, Groningen, Netherlands.

20 gLaboratory of Experimental Hematology, Faculty of Medicine and Health Sciences, Vaccine and 21 Infectious Disease Institute (VAXINFECTIO), University of Antwerp, Antwerp, Belgium. 
${ }^{\mathrm{h}}$ Laboratory of Neurochemistry and Behavior, Department of Biomedical Sciences, Institute BornBunge, University of Antwerp, Antwerp, Belgium; and Department of Neurology, Memory Clinic of Hospital Network Antwerp (ZNA) Middelheim and Hoge Beuken, Antwerp, Belgium; and Department of Neurology and Alzheimer Research Center, University of Groningen and University Medical Center Groningen, Groningen, Netherlands.

27 iSociety for Brain Mapping \& Therapeutics, Brain Mapping Foundation, Pacific Palisades, CA and T-Neuro Pharma, Inc., Albuquerque, NM \& Aptos, CA, USA.

29

*These authors contributed equally to the manuscript.

$31 \dagger$ Present addresses: RC, Dept. of Microbiology \& Molecular Biology, Brigham Young University, Provo, UT, United States; AM, Dept. of Translational Science, A2 Biotherapeutics, Inc., 30301 Agoura Road \#210, Agoura Hills, CA, 91301. 


\section{Summary}

Sporadic Alzheimer's disease, the most common neurodegenerative disorder of aging, is characterized by cerebral plaques and neurofibrillary tangles. Experimental rodents develop plaques but neither tangles nor substantial neurodegeneration under conditions that guarantee Alzheimer's in humans ${ }^{1}$, suggesting rodents lack critical co-initiation factors. Accumulation of antigen-reactive memory $\operatorname{CD8} \mathrm{T}$ cells increases with aging ${ }^{2}$, and was recently revealed as a hallmark of human Alzheimer's ${ }^{3}$. The impact of this process on disease initiation, however, has not been established because age-related $T$ cell changes are muted in rodents ${ }^{4-6}$. We developed a mouse model of human-like CD8 $\mathrm{T}$ cell aging that promotes antigen-reactive memory $\mathrm{CD8} \mathrm{T}$ cell accumulation ${ }^{7}$. Here we show that these "hiT" mice develop all major hallmarks of Alzheimer's with aging, including tangle-like inclusions and substantial neurodegeneration. Antigen-reactive CD8 $\mathrm{T}$ cells analogous to those in ${ }^{\text {hiT }}$ mice increased in Alzheimer's brain, but decreased earlier in blood, where their loss effectively distinguished the Alzheimer's continuum from aging controls. Our findings establish a clinically relevant mouse model for sporadic Alzheimer's and show that agerelated immune dysfunction critically contributes to its initiation. They also identify useful immune-based targets to track and potentially treat human Alzheimer's, while validating a model system to examine age-related disease immuno-biology more generally. 


\section{Results}

\section{Generation of "hi T" cells in nude mice}

We previously demonstrated that young B6.Foxn1 (B6.nude) mice injected with purified donor B6 CD8 T cells rapidly develop a T cell compartment dominated by homeostatically induced, selfreactive $\mathrm{CD} 8 \mathrm{~T}$ cells with a resident memory phenotype (hi $\mathrm{T}_{\mathrm{RM}}$ ) identical to age-related CD8 $\mathrm{T}$ cells accumulating in affected aged mice. This mirrors the dominance of circulating age-related memory CD8 T cells in moderate- to old-age humans ${ }^{8}$, and rendered selected APP-reactive memory CD8 T cell levels in mice similar to those in aging humans (Extended Data Fig. E1) ${ }^{9}$.

${ }^{\text {hi }} \mathrm{T}_{\mathrm{RM}}$ recipient mice also exhibited age-related tissue pathology, including neuroinflammation and increased memory CD8 T cells in brain, along with other factors associated with $\mathrm{AD}$. We therefore examined whether B6.Foxn1 ${ }^{\text {hi }} \mathrm{T}_{\mathrm{RM}}$ recipients exhibited additional neuropathological features of $\mathrm{AD}$ various times after $\mathrm{T}$ cell injection (Extended Data Fig. E2a). To ensure our observations were due to functional rather than purely physical aspects of $\mathrm{CD} 8{ }^{\text {hi }} \mathrm{T}_{\mathrm{RM}}$ accumulation in the brain, we included B6.Foxn1 cohorts injected with PBS, and with CD8 T cells from wild-type, Perforin 1deficient, or IFN $\gamma$-deficient donors, (PBS, wt-CD8, PrfKO-CD8, and Ifn $\gamma$ KO-CD8 groups, respectively).

\section{Aß and neurofibrillary deposition}

CD8 T cells expanded in circulation of all B6.Foxn1 recipients ${ }^{7}$. By contrast, Amyloid Precursor Protein (APP) and its cleavage products including A $\beta$ were dramatically increased only in brains of wt-CD8 group mice 3 weeks after injection (Fig. 1). Detergent-soluble A $\beta 1-40$, but not A $\beta 1$ 42, was also elevated 10 weeks post-injection in ELISA analysis, and Western blot confirmed 
prominent involvement of hippocampus at this time point (Fig. 1a, b; Extended Data Fig. E2b). By 6 months post-injection, increased $A \beta$ deposition in brain vasculature was evident in wt-CD8 group mice, consistent with selective elevation of A $\beta 1-40$ (Extended Data Fig. E2c). At 15 months post-injection, $A \beta 1-40$ was still significantly elevated in wt-CD8 brain (Fig. 1b), and A $\beta$ plaques were evident in entorhinal cortex, hippocampus, and cingulate cortex of wt-CD8 and Ifn $\gamma \mathrm{KO}-\mathrm{CD} 8$ groups (Fig. 1c, d). Unlike mice expressing familial gene mutations found in human AD, A $\beta$ plaques in both these groups were mainly diffuse and detergent-soluble (Extended Data Fig. E3a, b), with little co-staining by curcumin or ThioS (Fig. 1c; Extended Data Fig. E4a, b). The discrete amyloid pathology in the wt-CD8 group encouraged examination of additional AD-associated features such as tau phosphorylation and aggregation.

Detergent-soluble phospho-tau (pTau) was slightly (30\%) but significantly increased by 10 weeks post-injection in wt-CD8 group forebrain, while pTau paired helical filaments (PHFs, which mature to form NFTs in AD) were increased nearly 5-fold (Fig. 1e). Soluble pTau did not remain elevated 15 months post-injection, however, while PHFs remained significantly elevated, but at lower than earlier levels (2.5-fold decreased; Fig. 1f). We speculated this could be due conversion of pTau isoforms to more insoluble aggregated species after 10 weeks. Indeed, fibril-staining reagents, including curcumin and Thio-S, revealed cellular inclusions within wt-CD8 group hippocampus 6 months post-injection (Extended Data Fig. E4a, b). These inclusions were apparent before larger amyloid deposits appeared, although small plaques were occasionally associated with them (Extended Data Fig. E4a, b), and were absent from aged AD-transgenic mice, as well as from AD-transgenic rats that reportedly accumulate PHFs (Extended Data Fig. E4a, b) ${ }^{10}$. 
Gallyas staining also revealed discrete silver-stained cellular structures in wt-CD8 and Ifn $\gamma \mathrm{KO}-$ CD8 hippocampus and cortex 15 months post-injection, that were not seen in AD-transgenic mice (Fig. 1g, h). These structures appeared similar to NFTs from human AD patients (Extended Data Fig. E5a, b). In addition, sequential staining revealed that Gallyas-stained structures in wt-CD8 and Ifn $\gamma \mathrm{KO}-\mathrm{CD} 8$ groups were derived from $\mathrm{pTau}^{+}$neurons with intact nuclei (Fig. 1g), and that Gallyas and pTau staining was superimposable and distinct from that of A $\beta$ (Fig. 1g; Extended Data Fig. E5c). "Ghost tangles”, NFTs in dead neurons that are often present in human AD 11,12, were not observed. These data suggest that hi $\mathrm{T}_{\mathrm{RM}}$ promote the coordinated deposition of parenchymal A $\beta 40$, diffuse plaques, and fibrillar pTau inclusions in live neurons, either directly or by indirectly promoting neuroinflammation.

\section{Immune \& neuroinflammatory infiltration}

Our previous work established that astrogliosis, microgliosis, and CD8 T cell brain infiltration were all increased in wt-CD8 group ${ }^{\text {hi }} \mathrm{T}_{\mathrm{RM}}$ hosts ${ }^{7}$. We therefore examined the relationship of observed neuroinflammatory features to $A \beta$ plaque burden to determine the immune population most directly associated with neuropathology. A $\beta$ plaque burden correlated strongest with hippocampal CD8 T cell numbers compared to astrogliosis or microgliosis, consistent with a more direct impact of adaptive than innate immune cells on amyloid pathology (Extended Data Fig. E6). In this context, it is intriguing that, while PrfKO-CD8 group mice failed to exhibit either CD8 T cells in brain, or any significant AD-like neuropathology, Ifn $\gamma \mathrm{KO}-\mathrm{CD} 8$ group mice retained significantly increased plaques and NFT-like structures in hippocampus and entorhinal cortex, but not in cingulate cortex (Fig. 1d, h). This resembles the distribution of protein aggregates early in human $\mathrm{AD}{ }^{13}$, and as such suggests that neuroinflammation hastens AD-like neuropathology in 
118

119

120

121

122

123

124

125

126

127

128

129

130

131

132

133

134

${ }^{\text {hi }} T_{R M}$ mice, but is not required for its development. Taken together, our data suggest that ${ }^{\text {hi }} T_{R M}$ may directly promote pathologic features of AD-like neurodegeneration.

\section{Neuronal loss \& cerebral atrophy}

Robust neurodegeneration is not present in mouse $\mathrm{AD}$ models without addition of transgenes uninvolved in human $\mathrm{AD}^{1,14}$. To determine if overt neurodegeneration was present in ${ }^{\text {hi }} \mathrm{T}_{\mathrm{RM}}$ mice, we stained and counted $\mathrm{NeuN}^{+}$neurons in $\mathrm{CA} 1, \mathrm{CA} 2$, and $\mathrm{CA} 3$ of hippocampus, assessed brain mass, and quantified Western blots of NeuN and synaptic proteins. Loss of $\mathrm{NeuN}^{+}$cells in wt-CD8 group mice was visually apparent in hippocampal immunostains, and was verified by $\mathrm{NeuN}^{+}$cell counts at 15 months post-injection (Fig. 2a-c). Loss of brain mass was also evident in wt-CD8 group, and progressed from 5\% at 6 months, to $10 \% 15$ months post-injection (Fig. 2d), which is comparable to terminal brain atrophy in human $\mathrm{AD}^{15}$. Western blots confirmed an approximate $10 \%$ decrease in NeuN, as well as in the synaptic protein, Drebrin, and a non-significant trend toward lower Synaptophysin protein, 15 months post-injection (Fig. 2e, f). Importantly, loss in brain mass correlated with decreased $\mathrm{NeuN}$ across all $\mathrm{T}$ cell injection groups, establishing a direct relationship between brain mass and neuronal loss (Fig. 2g). Thus, B6.nude ${ }^{\text {hi }} \mathrm{T}_{\mathrm{RM}}$ recipients exhibited robust and easily discernible neurodegeneration by multiple measures, with related brain atrophy. This revealed the possibility that ${ }^{\text {hi }} \mathrm{T}_{\mathrm{RM}}$ mice might exhibit AD-like dementia as well.

\section{Severe cognitive impairment}

Prior to cognitive testing in ${ }^{\text {hi }} \mathrm{T}_{\mathrm{RM}}$ mice, we established that spontaneous locomotor activity was not significantly different between treatment and control groups in Open Field testing 3, 6, and 13 months after T cell injection (Fig, 3a; Extended Data Fig. E7a), ruling out motor deficits such as those in multiple sclerosis. Nevertheless, all groups exhibited motor deficits that increased with 
age, but this was unrelated to treatment. In contrast to motor activity, Fear Conditioning (FC) response to contextual but not cued learning was reduced in wt-CD8 group mice 6 months after T cell injection, with both contextual and cued learning impaired in the same mice tested 5 months later (Fig 3b). The hippocampus is required for contextual FC responses, whereas both hippocampus and amygdala are required for cued FC responses. Hence, the FC results suggest that ${ }^{\text {hi }} \mathrm{T}_{\mathrm{RM}}$ mediate damage to hippocampus alone early on, and cause further damage to amygdala later, a pattern commonly seen in human $\mathrm{AD}{ }^{16}$. Contextual performance at 6 and 11 months also correlated with brain mass (Extended Data Fig. E7b), further underscoring the relationship of cognitive decline to physical neurodegeneration.

Spontaneous Alternation Behaviour (SAB) 12 months post-injection independently confirmed behavioral abnormalities in wt-CD8 group mice exclusively. This test is based on the preference of mice to alternately explore two alleys, which requires working memory of the alley previously entered. The lowest possible score of 50\% indicates random alley choice, reflecting either no working memory, or complete lack of preference. The 55-56\% SAB score in the PBS group was comparable to published wild-type values ${ }^{17}$, but the wt-CD8 group SAB was significantly lower at $50 \%$ (Fig. 3c). To verify whether this reflected loss of working memory or lack of preference, we employed the Barnes Maze test at 14 months, a more focused measure of hippocampusdependent learning and memory. In contrast to all other groups, wt-CD8 mice showed no ability to learn the maze over the initial 4-day training period, indicating a profound learning and memory deficit (Fig. 3d). Given this inability, wt-CD8 mice were uniquely impaired on subsequent memory retention and reversal phases of the maze as well (Fig. 3e-g). As with Fear-Conditioning, latency to solve the Barnes Maze correlated inversely with brain mass (Extended Data Fig. E7c). Taken 
162 together, these tests suggest that fully functional ${ }^{\text {hi }} \mathrm{T}_{\mathrm{RM}}$ mediate severe, progressive impairment of hippocampus-dependent learning and memory, but not locomotor activity.

Because cognitive impairment is differentially associated with amyloid and tau pathology in AD, we further addressed whether cognitive loss was associated with $\mathrm{A} \beta$ and/or pTau metrics in ${ }^{\text {hi }} \mathrm{T}_{\mathrm{RM}}$ mice. Poor performance on Barnes Maze (total latency below median = $\mathrm{BM}^{\mathrm{lo}}$ ) exhibited significant association only with increased pTau PHFs on Western blots (Extended Data Fig. E7d), and not with Triton-soluble or GuanidineHCl-soluble $A \beta 1-40$ or A $\beta 1-42$ on ELISA, or with detergent soluble pTau by Western blot (Extended Data Fig. E7e, f). Thus, cognitive impairment appeared preferentially associated with fibrillar tau pathology in wt-CD8 group mice, as it is in human AD 18 .

\section{${ }^{\text {hi}}$ T cell metrics in Alzheimer's disease}

To examine possible involvement of $\mathrm{T}$ cells analogous to those in ${ }^{\text {hi }} \mathrm{T}_{\mathrm{RM}}$ mice in $\mathrm{AD}$, we quantified CD8 T cells in blood, their relationship to cognitive decline, and their presence in brain, using three human cohorts (Fig. 4a). We first examined KLRG1 ${ }^{+}$and KLRG1- CD8 T cell subpopulations in blood from aging control subjects (CTRL), MCI patients with an AD-typical CSF biomarker profile (MCI-AD), MCI patients without an AD-typical CSF biomarker profile (MCI), and sporadic AD patients (AD) (Cohort 1). KLRG1 ${ }^{+}$CD8 T cells were not significantly increased in AD blood (Fig. 4b), but increased in rough correlation with age, while KLRG1' CD8 T cells did not (Extended Data Fig. E8). In contrast, KLRG1 ${ }^{+} \mathrm{CD} 8 \mathrm{~T}$ cells co-stained with pHLAA2 multimers to a human $\mathrm{T}$ cell epitope analogous to that recognized by $\mathrm{T}$ cells in ${ }^{\mathrm{hi}} \mathrm{T}_{\mathrm{RM}}$ mice $\left[\mathrm{APP}_{(471-479)}\right]$ were dramatically decreased in the blood of MCI, MCI-AD, and AD patients (Fig. 4c, d). While this differs from other (i.e., EBV-specific) CD8 $\mathrm{T}$ cells that increase in $\mathrm{AD}^{3}$, 
segregation of CTRL and MCI patients by cognitive performance score did reveal that KLRG1 ${ }^{+}$ CD8 T cells increased during age-related cognitive decline (Fig. 4d). Moreover, $\operatorname{APP}_{(471-479)-}$ specific KLRG1 ${ }^{+}$CD8 $\mathrm{T}$ cell levels correlated significantly with cognitive decline but not age itself (Fig. 4e). The parental KLRG1 ${ }^{+} \mathrm{CD} 8 \mathrm{~T}$ cell population thus appears to expand in blood earlier than other memory CD8 $\mathrm{T}_{\text {cells }}{ }^{3}$, and appears to contract as cognitive decline is clinically diagnosed. This could conceivably prompt compensatory expansion by cells such as $\mathrm{T}_{\text {EMRA }}$ to occupy a depleted memory CD8 T cell pool (Extended Data Fig. E8).

Decreased $\mathrm{KLRG}^{+}{ }^{+} \mathrm{APP}_{(471-479)}$-specific $\mathrm{CD} 8 \mathrm{~T}$ cells in blood distinguished AD from normal aging patients with less overlap than another promising blood-based biomarker for $\mathrm{AD}$ in development, plasma levels of P-Tau217 (Extended Data Fig. E9a, b) ${ }^{19}$. Diagnostic potential of this decrease was indicated in Receiver Operating Characteristic (ROC) analysis, where it effectively distinguished AD and AD-related MCI from CTRL blood (Extended Data Fig. E9c). Specifically, area under the curve (AUC) for AD-related MCI and AD were 0.865 and 0.892 , respectively, with AD-unrelated MCI AUC $=0.760$ (Extended Data Fig. E9c).

We next examined CD8 and Perforin-1 content in AD brain. Importantly, Western blots rendered the expected antibody specificities (68-75 $\mathrm{kDa}$ Prf1; 33-35 kDa CD8 $\alpha$ ), with anti-Prf1 staining lymphocytic nuclei with the expected punctate pattern (Fig. 4f). Perforin1 and CD8 Western signals were correlated $(\mathrm{n}=6 ; \mathrm{r}=0.8155, P=0.048$; not shown), and both were increased in AD cortex, with Perforin1 reaching statistical significance (Fig. 4g). This is consistent with elevated cytolytic CD8 T cells in AD brain. To examine this more directly, we stained hippocampal sections from AD patients and normal aging controls by tissue immunofluorescence and quantified cells stained with anti-CD8 alone or co-stained with $\mathrm{APP}_{(471-479)}$-/HLA-A2 multimers (Fig. 4 h). As in ${ }^{\text {hi }} \mathrm{T}_{\mathrm{RM}}$ mice, total CD8 $\mathrm{T}$ cells were not significantly elevated in $\mathrm{AD}$ brain $(\mathrm{n}=10 ; 1.6 \pm 0.29$ vs. 
$2.3 \pm 0.55, P=0.31$; not shown). Nevertheless, APP-specific CD8 T cells were increased in

208 perivasculature and cortical regions of $\mathrm{AD}$ hippocampus (Fig. 4i), similar to ${ }^{\text {hi }} \mathrm{T}_{\mathrm{RM}}$ mice and as

209 predicted by that model.

210 Conclusion

211 In conclusion, we show for the first time that all major hallmarks of human AD can be elicited by

212 a single inductive event in mice. This pathology is dependent on expansion in blood and entry into

213 brain of age-related resident memory CD8 $\mathrm{T}$ cells $\left(\mathrm{CD} 8 \mathrm{~T}_{\mathrm{RM}}\right)$, and exhibits gross similarity to

214 human $\mathrm{AD}$ with important distinctions. Notably, amyloidopathy in ${ }^{\text {hi }} \mathrm{T}_{\mathrm{RM}}$ mice was limited to $\mathrm{A} \beta 1$ -

21540 and mainly diffuse plaques, unlike the $\mathrm{A} \beta 1-42$ and mature neuritic plaques that predominate in

216 many AD patients. Moreover, while ${ }^{\text {hi }} \mathrm{T}_{\mathrm{RM}}$ mice exhibited fibrillar NFT-like structures in live cells,

217 they did not harbor the ghost tangles in dead neurons often seen in human AD. While $\mathrm{A} \beta$

218 differences could reflect deficiencies in $A \beta 1-42$ clearance and/or fibril formation characteristic of

219 rodent brains ${ }^{20,21}$, the lack of ghost tangles is likely due to decreased expression in adult mice of

220 the ghost tangle-promoting isoforms of MAPT, the gene encoding tau proteins ${ }^{11,12}$.

222 Despite these distinctions, ${ }^{\text {hi }} \mathrm{T}_{\mathrm{RM}}$ mice exhibited unique similarities to sporadic $\mathrm{AD}$ in humans beyond amyloidosis, fibrillar tauopathy, and robust neurodegeneration. These included cognitive

224 decline that initiated with hippocampus-dependent tasks with later progression to amygdala225 dependent tasks ${ }^{16}$; significant association of cognitive loss exclusively with fibrillar tau pathology

$22{ }^{18}$; neuroinflammation that exacerbated neuropathology ${ }^{22}$; and accumulation of antigen-specific

227 memory CD8 T cells in brain ${ }^{7}$, the most recently characterized hallmark of human $\mathrm{AD}^{3}$. The 228 pattern of ample NFT-like structures, vascular amyloidosis, A $\beta 1-40$ and diffuse plaque 
predominance seen in ${ }^{\text {hi }} \mathrm{T}_{\mathrm{RM}}$ mice also resembled that of at least one subpopulation of human $\mathrm{AD}$ patients in carriers of the APP Iowa mutation ${ }^{23,24}$.

CD8 $\mathrm{T}$ cells reactive to an antigenic epitope nearly identical to that recognized by brain-localized $\mathrm{T}$ cells in ${ }^{\text {hi }} \mathrm{T}_{\mathrm{RM}}$ mice, were increased in $\mathrm{AD}$ brain but decreased in $\mathrm{AD}$ and MCI blood, suggesting that their movement from blood to brain is involved in neuropathology. Consistent with this notion, decreased levels of $\mathrm{KLRG}^{+} \mathrm{APP}_{(471-479)} / \mathrm{HLA}-\mathrm{A} 2$ multimer-binding CD8 $\mathrm{T}$ cells in blood correlated with cognitive impairment in MCI patients. While somewhat reminiscent of a distinct subpopulation of CD8 effector-memory $\mathrm{T}$ cells, $\mathrm{T}_{\mathrm{EMRA}},{ }^{\mathrm{hi}} \mathrm{T}_{\mathrm{RM}}$ analogues are distinct in that they were weakly age-related, their decrease rather than increase correlated with cognitive loss, and were prominently reactive to self antigen ${ }^{3}$. TEMRA are also absent from mice ${ }^{3,25,26}$. Further contrasting these subpopulations. Finally, CD8 $\mathrm{T}_{\mathrm{RM}}$ expanded during age-related cognitive decline, a stage that precedes $\mathrm{MCI}$ when $\mathrm{T}_{\mathrm{EMRA}}$ are reported to expand. Thus, $\mathrm{CD} 8 \mathrm{~T}_{\mathrm{RM}}$ alterations occur earlier are unique with respect to those of $\mathrm{T}_{\mathrm{EMRA}}$, and may be more direct beacons of $\mathrm{AD}$ induction than other immune or non-immune factors in blood.

Accordingly, levels of KLRG1 ${ }^{+}$HLA-A2/APP(471-479)-specific CD8 T cells in blood distinguished AD from normal aging patients with significantly less overlap than plasma P-Tau217, a bloodbased biomarker met with much enthusiasm (Extended Data Fig. E9a, b) ${ }^{19}$, and tracked AD and AD-related MCI with high accuracy (Extended Data Fig. E9c). Further assessment of the potential of hi $\mathrm{T}_{\mathrm{RM}}$ metrics as diagnostic biomarkers for $\mathrm{AD}$ requires multi-center validation, as well as prospective and longitudinal analyses with non-AD dementia cohorts. From a therapeutic perspective, it will be equally important to determine whether modulation of antigen-specific ${ }^{\text {hi }} \mathrm{T}_{\mathrm{RM}}$ presence or function can alter the course of $\mathrm{AD}$-like neurodegeneration in mice, and determine if effective treatments for $\mathrm{AD}$ critically modulate these cells in humans. Finally, continued 
252 examination of hi $\mathrm{T}_{\mathrm{RM}}$ mice on multiple strain backgrounds, and harboring various risk factors for $253 \mathrm{AD}$ and distinct age-related disorders, may lead to a more comprehensive understanding of their 254 biology, genetics, and immune features. 


\section{References:}

2581 Schwab, C., Hosokawa, M. \& McGeer, P. L. Transgenic mice overexpressing amyloid beta protein are an incomplete model of Alzheimer disease. Exp Neurol 188, 52-64 (2004).

2602 Ritzel, R. M. et al. Age-Associated Resident Memory CD8 T Cells in the Central Nervous 261 System Are Primed To Potentiate Inflammation after Ischemic Brain Injury. J Immunol 196, 3318-3330, doi:10.4049/jimmunol.1502021 (2016).

2633 Gate, D. et al. Clonally expanded CD8 T cells patrol the cerebrospinal fluid in Alzheimer's disease. Nature 577, 399-404, doi:10.1038/s41586-019-1895-7 (2020).

2654 Clambey, E. T., van Dyk, L. F., Kappler, J. W. \& Marrack, P. Non-malignant clonal 266 expansions of CD8+ memory $\mathrm{T}$ cells in aged individuals. Immunol Rev 205, 170-189 (2005).

2685 Clambey, E. T., White, J., Kappler, J. W. \& Marrack, P. Identification of two major types of age-associated CD8 clonal expansions with highly divergent properties. Proc Natl Acad Sci U S A 105, 12997-13002 (2008).

2716 den Braber, I. et al. Maintenance of peripheral naive T cells is sustained by thymus output 272 in mice but not humans. Immunity 36, 288-297, doi:10.1016/j.immuni.2012.02.006 (2012).

2737 Panwar, A. et al. Functional recreation of age-related CD8 T cells in young mice identifies drivers of aging- and human-specific tissue pathology. Mech Ageing Dev 191, 111351, doi:10.1016/j.mad.2020.111351 (2020).

2768 Schwab, R. et al. Expanded $\mathrm{CD}^{+}$and $\mathrm{CD}^{+} \mathrm{T}$ cell clones in elderly humans. $J$ Immunol 277 158, 4493-4499 (1997). 
2789 Nikolich-Zugich, J., Li, G., Uhrlaub, J. L., Renkema, K. R. \& Smithey, M. J. Age-related changes in CD8 T cell homeostasis and immunity to infection. Semin Immunol $\mathbf{2 4}, 356-$ 364, doi:10.1016/j.smim.2012.04.009 (2012).

28110 Cohen, R. M. et al. A transgenic Alzheimer rat with plaques, tau pathology, behavioral doi:10.1523/jneurosci.3672-12.2013 (2013).

11 Lace, G. et al. Hippocampal tau pathology is related to neuroanatomical connections: an ageing population-based study. Brain : a journal of neurology 132, 1324-1334, doi:10.1093/brain/awp059 (2009).

12 Uchihara, T., Hara, M., Nakamura, A. \& Hirokawa, K. Tangle evolution linked to differential 3- and 4-repeat tau isoform deposition: a double immunofluorolabeling study using two monoclonal antibodies. Histochem Cell Biol 137, 261-267 (2012).

13 Reitz, C., Honig, L., Vonsattel, J. P., Tang, M. X. \& Mayeux, R. Memory performance is related to amyloid and tau pathology in the hippocampus. Journal of neurology, neurosurgery, and psychiatry 80, 715-721, doi:10.1136/jnnp.2008.154146 (2009).

14 Codita, A., Winblad, B. \& Mohammed, A. H. Of mice and men: more neurobiology in dementia. Curr Opin Psychiatry 19, 555-563 (2006).

15 Sluimer, J. D. et al. Whole-brain atrophy rate and cognitive decline: longitudinal MR study of memory clinic patients. Radiology 248, 590-598 (2008).

16 Serrano-Pozo, A., Frosch, M. P., Masliah, E. \& Hyman, B. T. Neuropathological alterations in Alzheimer disease. Cold Spring Harbor perspectives in medicine 1, a006189, doi:10.1101/cshperspect.a006189 (2011). 
30017 Ma, Q. L. et al. Beta-amyloid oligomers induce phosphorylation of tau and inactivation of

301

302

303

304

305

306

307

308

309

310

311

insulin receptor substrate via c-Jun N-terminal kinase signaling: suppression by omega-3 fatty acids and curcumin. J Neurosci 29, 9078-9089 (2009).

18 Brier, M. R. et al. Tau and Abeta imaging, CSF measures, and cognition in Alzheimer's disease. Science translational medicine 8, 338ra366, doi:10.1126/scitranslmed.aaf2362 (2016).

19 Palmqvist, S. et al. Discriminative Accuracy of Plasma Phospho-tau217 for Alzheimer Disease vs Other Neurodegenerative Disorders. JAMA 324, 772-781, doi:10.1001/jama.2020.12134 (2020).

20 Banks, W. A., Robinson, S. M., Verma, S. \& Morley, J. E. Efflux of human and mouse amyloid beta proteins 1-40 and 1-42 from brain: impairment in a mouse model of Alzheimer's disease. Neuroscience 121, 487-492 (2003).

21 Shin, R. W. et al. Amyloid beta-protein (Abeta) 1-40 but not Abeta1-42 contributes to the experimental formation of Alzheimer disease amyloid fibrils in rat brain. J Neurosci 17, 8187-8193 (1997).

22 Liang, K. Y. et al. Exercise and Alzheimer's disease biomarkers in cognitively normal older adults. Ann Neurol 68, 311-318, doi:10.1002/ana.22096 (2010).

23 Grabowski, T. J., Cho, H. S., Vonsattel, J. P., Rebeck, G. W. \& Greenberg, S. M. Novel amyloid precursor protein mutation in an Iowa family with dementia and severe cerebral amyloid angiopathy. Ann Neurol 49, 697-705 (2001).

24 Hellstrom-Lindahl, E., Viitanen, M. \& Marutle, A. Comparison of Abeta levels in the brain of familial and sporadic Alzheimer's disease. Neurochemistry international 55, 243-252, doi:10.1016/j.neuint.2009.03.007 (2009). 
25 Pangrazzi, L. et al. Increased IL-15 Production and Accumulation of Highly Differentiated CD8(+) Effector/Memory T Cells in the Bone Marrow of Persons with Cytomegalovirus. Frontiers in immunology 8, 715, doi:10.3389/fimmu.2017.00715 (2017).

26 Willinger, T., Freeman, T., Hasegawa, H., McMichael, A. J. \& Callan, M. F. Molecular signatures distinguish human central memory from effector memory CD8 T cell subsets. $J$ Immunol 175, 5895-5903, doi:10.4049/jimmunol.175.9.5895 (2005).

27 West, M. J., Slomianka, L. \& Gundersen, H. J. G. Unbiased stereological estimation of the total number of neurons in the subdivisions of the rat hippocampus using the optical fractionator. The Anatomical Record 231, 482-497, doi:10.1002/ar.1092310411 (1991).

28 Bach, M. E., Hawkins, R. D., Osman, M., Kandel, E. R. \& Mayford, M. Impairment of spatial but not contextual memory in CaMKII mutant mice with a selective loss of hippocampal LTP in the range of the theta frequency. Cell 81, 905-915, doi:10.1016/00928674(95)90010-1 (1995).

29 Pitts, M. W. Barnes Maze Procedure for Spatial Learning and Memory in Mice. Bioprotocol 8, doi:10.21769/bioprotoc.2744 (2018).

30 Engelborghs, S. et al. Diagnostic performance of a CSF-biomarker panel in autopsy$\begin{array}{lllll}\text { confirmed } & \text { dementia. } & \text { Neurobiol }\end{array}$ doi:10.1016/j.neurobiolaging.2007.02.016 (2008).

31 Petersen, R. C. Mild cognitive impairment as a diagnostic entity. Journal of internal medicine 256, 183-194, doi:10.1111/j.1365-2796.2004.01388.x (2004).

32 McKhann, G. M. et al. The diagnosis of dementia due to Alzheimer's disease: recommendations from the National Institute on Aging-Alzheimer's Association 
workgroups on diagnostic guidelines for Alzheimer's disease. Alzheimers Dement 7, 263269, doi:10.1016/j.jalz.2011.03.005 (2011).

34733 Dubois, B. et al. Advancing research diagnostic criteria for Alzheimer's disease: the IWG348 2 criteria. Lancet Neurol 13, 614-629, doi:10.1016/s1474-4422(14)70090-0 (2014).

349

350

351 

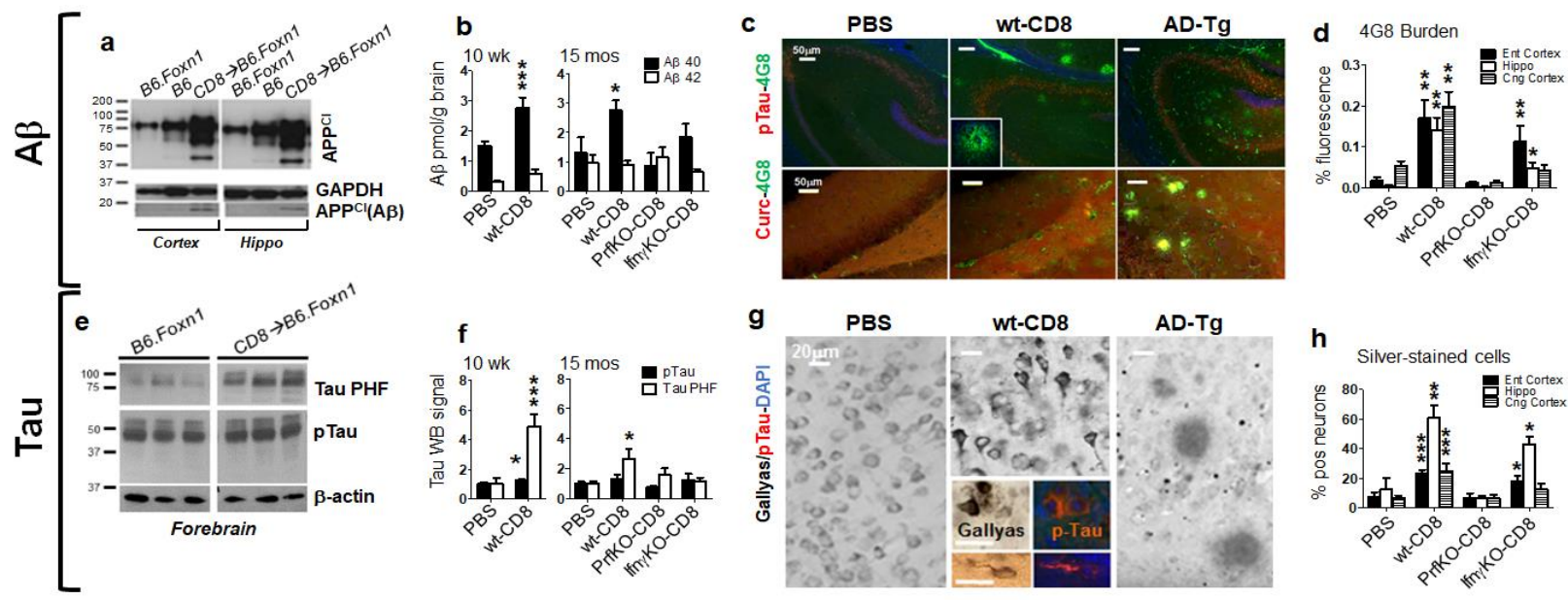

Fig. 1: Amyliod and pTau pathology. Western blots of APP cleavage products $\left(\mathrm{APP}^{\mathrm{Cl}}\right)$ in cortex, hippocampus 3 wk after injection $(\rightarrow)$ of recipients (a). B-J depict B6.Foxn1 recipients , 15 mos post-injection unless otherwise indicated. Forebrain A $\beta 1-40 / 42$ ELISA (b). Plaques \pm $358 \mathrm{pTau} /$ curcumin staining (c), and compiled 4G8 burden in entorhinal (Ent)/cingulate (Cng) cortex, 359 and hippocampus (Hippo) (d). Western blots (e), and compiled pTau and PHF signal. 360 Gallyas/silver-stained cells in ${ }^{\text {hi }} \mathrm{T}_{\mathrm{RM}}$ groups and 18-month-old $\mathrm{Tg} 2576$ (AD-Tg) mice, with 361 sequential pTau $\rightarrow$ Gallyas stains inset (g). Gallyas ${ }^{+}$neuron (h) percentages Plots depict averages $362 \pm$ SEM. $* P<0.05, * * P<0.01, * * * P<0.005$ by 2 -sided T-test, relative to PBS group. 

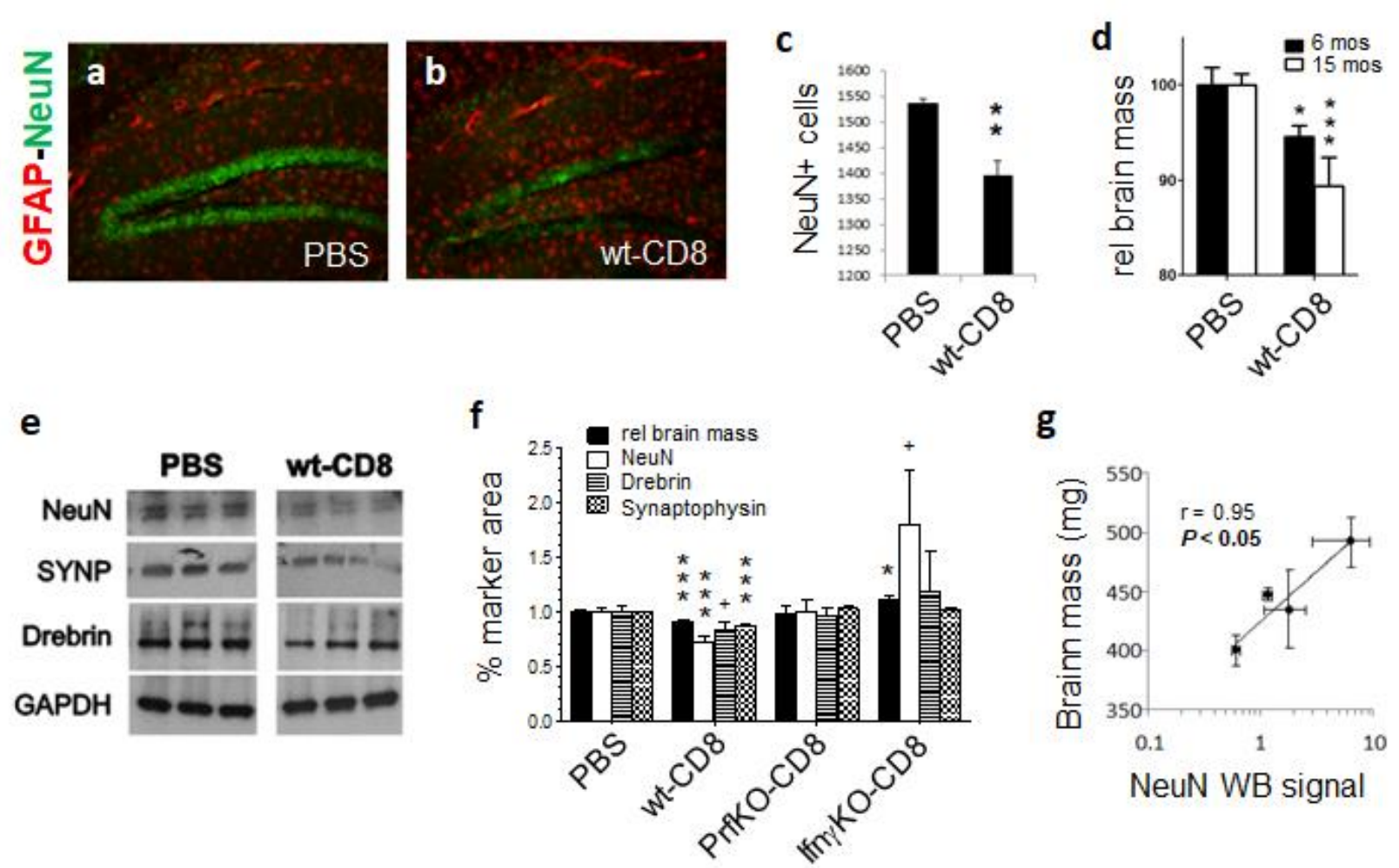

364 Fig. 2: Neurodegeneration in nude mice harboring hi $\mathbf{T}$ cells. Cell/control recipients in all panels 365 are B6.Foxn1 exclusively. NeuN and GFAP staining (a, b), and cell counts in hippocampus, 15 366 mos after cell/control injection (c). Brain atrophy over time in PBS and wt-CD8 groups (mass 367 normalized to PBS controls at each time point; d). Representative forebrain Westerns (e), and 368 GAPDH-normalized NeuN, Drebrin, and Synaptophysin Western signals (f). Correlation of NeuN 369 with brain weight (g). Plots depict averages \pm SEM. $* P<0.05, * * P<0.01, * * * P<0.005$ by 2 370 sided T-test, relative to PBS group. 

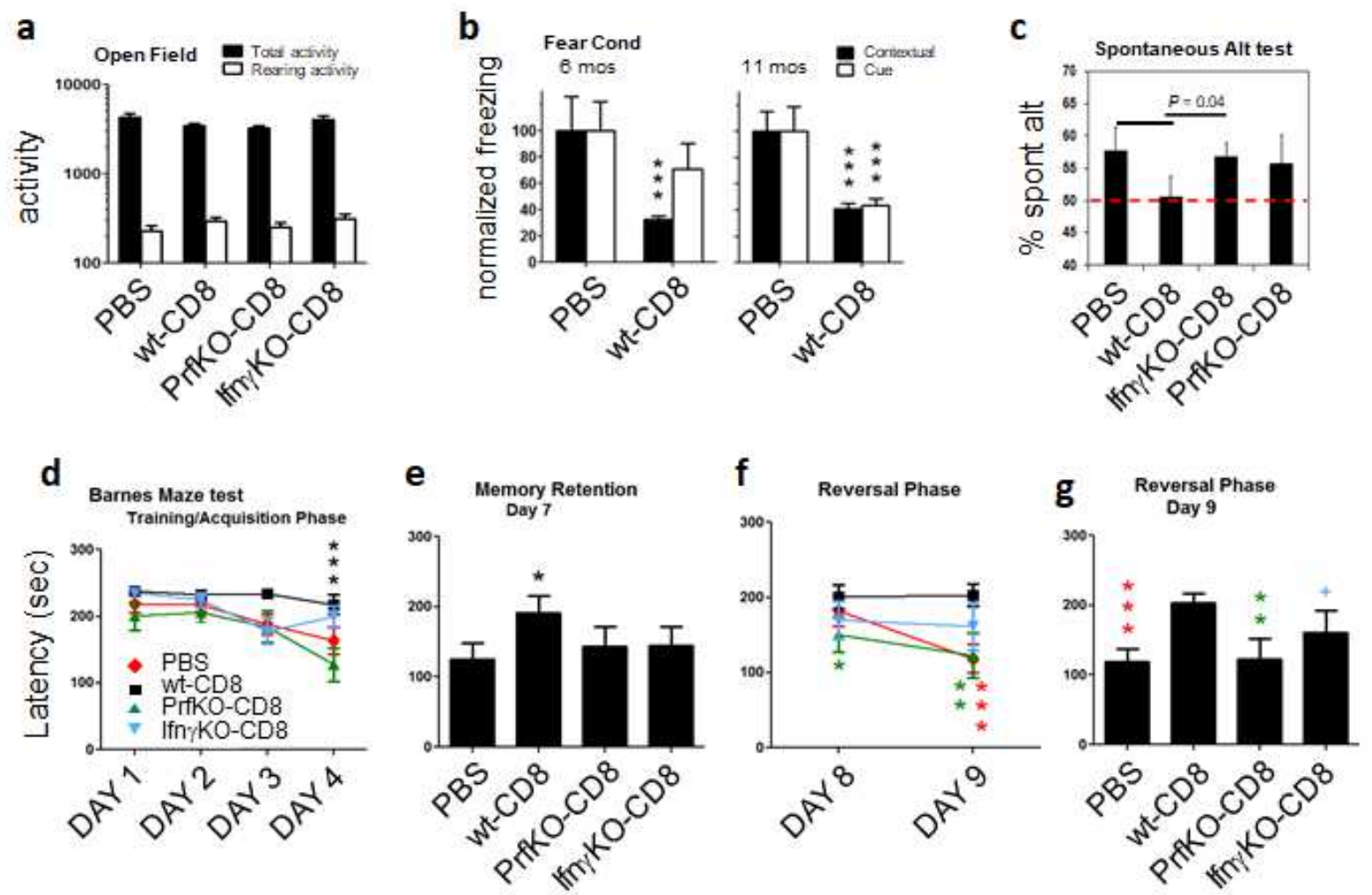

Fig. 3: Cognition in nude mice harboring hit cells. Representative Open Field test at 13 mos (a). Fear Conditioning over time (b), and Spontaneous Alternation Behavior at 12 months (c). Barnes Maze learning/training (d), retention (e), and reversal (f, g) phases, at 14 mos (black, colored symbols $=P$ relative to PBS, wt-CD8, respectively). Plots depict averages \pm SEM. $* P<$ $0.05, * * P<0.01, * * * P<0.005$ by 2 -sided ANOVA (panel d) or 2 -sided T-test (all others), relative to PBS group unless otherwise indicated. 
a
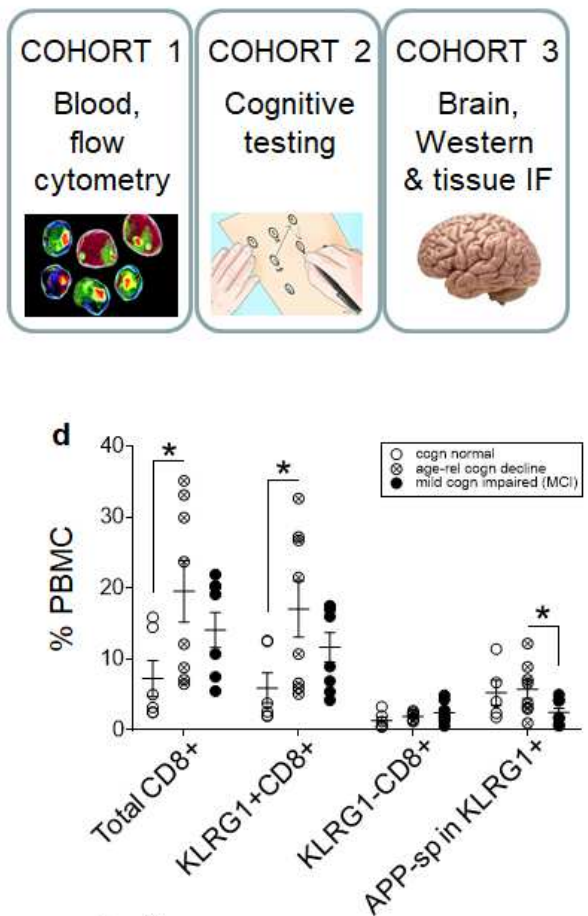
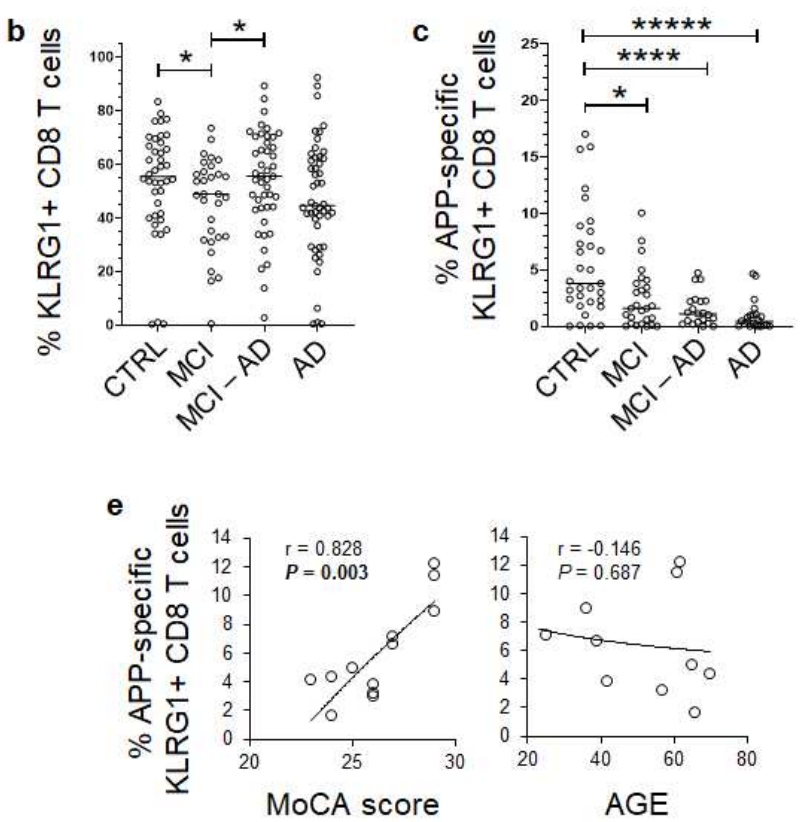
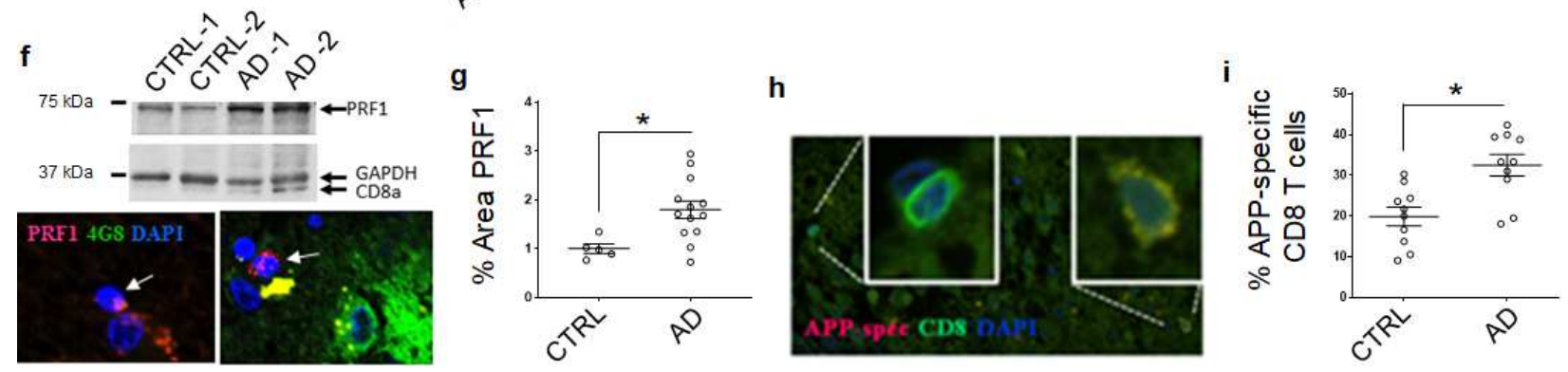

Fig. 4: hiT parameters in human Alzheimer's. Patient cohorts (a). KLRG1 ${ }^{+}$(b) and $\operatorname{APP}_{(471-}$ 479)/HLA-A2-reactive $\mathrm{KLRG1}^{+}$(c) CD8 T cells in CTRL, MCI \pm CSF AD biomarkers (MCI, MCIAD), and verified Alzheimer's (AD) blood. T cell subpopulations vs. MoCA score (d), and correlation of $\mathrm{APP}_{(471-479)} / \mathrm{HLA}-\mathrm{A} 2$-reactive $\mathrm{KLRG}^{+} \mathrm{CD} 8$ with score and age (e). PRF1 Western blot and immunofluorescence (f), with quantifications in age-matched CTRL and AD brains. (g). 
388 averages \pm SEM. $* P<0.05, * * P<0.01, * * * P<0.005, * * * * P<0.001$ by 2 -sided T-test, relative 389 to CTRL unless otherwise indicated. 


\section{Methods}

392

393

394

395

396

397

\section{Animal subjects}

Female C57BL/6, B6.Foxn1 mice, and congenic and/or syngeneic knockout strains (Jackson Labs) were housed in a pathogen-free vivarium under standard conditions on a 12-h light/12-h dark cycle with food and water ad libitum. Recipient animals were 8-10 week-old female B6.Foxn1, B6.Foxn1-AppKO, or B6.CD45.1-congenic mice; donors were 5-8 week-old females of the same strains. Specific numbers of animals used for all analytical methods are included in Supplemental Table 1. Cell derivation was randomized by pooling from $\geq 5$ donors per experiment. Young (8-10 wk) and aged (15 months) male and female C57BL/6 and B6.CD103-knockout mice (n = 12 young; $\mathrm{n}=7-8$ aged) were used to study age-related cognitive decline. Donor, recipient, and unmanipulated animals were maintained in a pathogen-free facility under the Cedars-Sinai Department of Comparative Medicine, with all breeding and genetic screening conducted at Jackson Laboratories (Bar Harbor, ME).

\section{Adoptive transfer of CD8 T cells}

Splenic $\mathrm{CD}^{+} \mathrm{T}$ cells from C57BL/6J female mice (5-7 weeks old) were purified using antiCD8 immunobeads (Miltenyi Biotech, Sunnyvale, CA). 3 x $10^{6}$ CD8 T cells in $50 \mu 1$ of PBS were injected intravenously into female C57BL/6J or B6.Foxn1 nude hosts. Transfer efficiency into B6.Foxn 1 hosts was validated by persistence of $\geq 5 \% \mathrm{CD} 8^{+} \mathrm{T}$ cells within splenic lymphocytes 3 weeks after injection. The order of treatments was randomized by alternating cell and control injections between individual recipients. For all subsequent analyses, performing investigators were blinded to both group definition and anticipated outcomes. 


\section{Tissue processing} 424 staining.

Brain and spleen were harvested from mice perfused with saline under deep anesthesia using a Ketamine and Xylazine (40-50 mg/kg i.p.) cocktail, until major organs such as liver and lungs lost color, and tissue was then excised for analysis. Upon removal of the whole brain from the cranium, the cerebellum, brainstem, and olfactory bulbs were removed, and remaining brain tissue was weighed on a Mettler balance for standardized brain mass assessment. Brains were sectioned 1 $\mathrm{mm}$ to the right of the longitudinal fissure (midline). Right hemispheres were flash frozen in $-80^{\circ} \mathrm{C}$ conditions for protein studies, followed by homogenization in Cell Lysis Buffer (Cell Signaling Technologies, MA), and centrifugation of nuclei. Cell lysates were separated into Triton-soluble, Sarkosyl-soluble and Sarkosyl-insoluble fractions using sequential incubations with 10\% (wt/V) salt sucrose solution and $1 \%(\mathrm{wt} / \mathrm{v})$ sarkosyl Salt Sucrose Solution. Left hemispheres were immersion fixed in $4 \%$ paraformaldehyde (duration?) and reserved for immunohistochemical

\section{Antibodies for tissue staining and western blot analyses}

Free-floating brain sections (8-14 $\mu \mathrm{m}$ thick) were mounted onto slides and blocked with Protein Block (Serum-Free, Dako, CA) for $1 \mathrm{~h}$ at RT. Sections were incubated at $4^{\circ} \mathrm{C}$ overnight with primary antibody in Protein Block (Dako, CA). Sections were rinsed 4x in PBS, and incubated $90 \mathrm{~min}$ in fluorochrome- or biotin-conjugated secondary antibody, with or without curcumin

430 (0.01\% in PBS), or with ThioS alone (1\% in PBS). Sections were washed, coverslipped, and 431 mounted with ProLongGold anti-fade media with DAPI (Invitrogen). Bright-field and fluorescent 432 images were obtained using a Zeiss AxioImagerZ1 with CCD camera (Carl Zeiss Micro imaging). 433 Image analysis of micrographs was performed with ImageJ (NIH). Anti-A $\beta / A P P$ antibody 
434 (ab14220, Abcam for 3-week time point; clone 4G8, Chemicon for all others) was used at 1:500

435 for immunohistochemistry (IHC) and 1:1000 for Western blot (WB). Anti-pTau pS199/202

436 antibody (Invitrogen) was used at 1:50 for IHC and 1:100 for WB, with PHFs confirmed with

437 Phospho-PHF-tau pSer202+Thr205 Antibody (AT8), used at 1:2000 for WB. Due to marker size,

438 pTau WB signal was normalized to that of $\beta$-actin (clone AC-74, Sigma), with GAPDH used for

439 normalization of all other markers. Anti-GFAP (Dako) was used at 1:250 for IHC and WB. Anti-

440 NeuN antibody (Chemicon) was used at 1:100 for IHC and WB. Anti-Iba1 (Wako, Ltd.) was used

441 at 1:200 for IHC. Anti-CD8 (clone 53-6.72, BD Pharmingen) was used at1:100 for IHC and 1:1000

442 for WB. All secondary antibodies (HRP, Alexa Flour-488, -594, -647; Invitrogen) were used at

443 1:200 for IHC and 1:2000 for WB. Multimer generation \& use: dextramers of established epitopes

444 for self/brain antigen $\left(\operatorname{Trp}-2-\mathrm{DCT}_{(180-188)} / \mathrm{H}-2 \mathrm{~K}^{\mathrm{b}}\right)$, and/or custom APP epitopes with predicted

445 affinities $<100 \mathrm{nM}\left(\right.$ NetMHC version 3.4; $\left.\mathrm{APP}(470-478) / \mathrm{H}-2 \mathrm{D}^{\mathrm{b}}\right)$, were manufactured by Immudex.

\section{Western blot for amyloid, tau, neural and immunological markers}

447

448

449

450

451

452

453

454
Triton-soluble cell lysates were electrophoretically separated on $12 \%$ Tris-HCl Precast Gels

(Bio-Rad), and blotted onto $0.2 \mu \mathrm{m}$ nitrocellulose membranes. Membranes were blocked with bovine serum albumin (BSA), incubated in sequential primary and secondary antibody dilutions for $1 \mathrm{hr}$ at room temperature with $\geq 3$ washes, developed with enhanced chemiluminescence substrate (GE Healthcare Biosciences; Pittsburgh, PA), and exposed onto Amersham Hyperfilm (GE Healthcare Biosciences; Pittsburgh, PA). 


\section{ELISA}

Supernatant from homogenized brain tissues was used for Triton-soluble A $\beta$. Insoluble pellets from Triton-homogenized brain were resuspended in 10 volumes $5 \mathrm{M}$ Guanidine $\mathrm{HCl} 4 \mathrm{hr}$ to generate Guanidine-soluble A $\beta$. Triton- and Guanidine-soluble samples were subjected to analysis by Soluble and Insoluble A $\beta$ ELISA (Invitrogen, Life Technologies; Grand Island, NY). Absorbance was read on a SPECTRAmax Plus384 microplate reader (Molecular Devices, Sunnyvale, CA) with data analyzed in Graphpad PRISM (Graphpad Software; San Diego, CA).

\section{Flow cytometry}

Purified $\mathrm{T}$ cells stained with respective Abs were analyzed by three-color flow cytometry (FACScan II; BD Biosciences, San Jose, CA) to assess purity. Antibodies were incubated with whole-spleen single-cell suspension in PBS with 5\% FBS, on ice for 30 min, followed by a wash with the PBS with 5\% FBS. Subsequently, 100,000-300,000 flow events were acquired.

\section{Gallyas silver staining}

Gallyas silver stain was used to visualize fibrillar aggregates. Free floating brain sections were placed in 5\% Periodic Acid for 3 min, washed twice and placed in Silver Iodide solution 1 min, followed by incubation in $0.5 \%$ Acetic Acid 5 min (2X), and rinsing with $\mathrm{dH}_{2} 0$. Sections were incubated in developer for $\sim 10$ min until sections were pale brown/gray, and stopped in $0.5 \%$ acetic acid for $5 \mathrm{~min}$, rinsed in $\mathrm{dH}_{2} \mathrm{O}$ and mounted. Stained sections were examined by microscopy. Stained neurons were counted from CA2 of hippocampus, and their proportions within total neurons visually quantified in triplicate from entorhinal and cingulate cortex. 


\section{Neuronal counts}

Whole-number neuronal estimates were performed using the optical fractionator method ${ }^{27}$ with stereological software (Stereo Investigator; MBF Bioscience). Para-median sagittal serial sections spaced $50 \mu \mathrm{m}$ apart were stained with NeuN. CA1, CA2, CA3 and other regions of interest were defined according to the Paxinos and Watson mouse brain atlas. A grid was placed randomly over the ROI, and cells were counted within three-dimensional optical dissectors (50 $\mu \mathrm{m} 50 \mu \mathrm{m}$ $10 \mu \mathrm{m}$ ) using a 100x objective. Within each dissector, $1 \mu \mathrm{m}$ guard zones at the top and bottom of section surface were excluded. Estimated totals weighted by section thickness were obtained with Stereo Investigator software, yielding a coefficient of error 0.10 .

\section{Behavioral testing - general}

Open Field testing was performed preceding all other behavioral tests, at 3, 6, and 13 months post-cell or -control injection. Testing order was randomized by alternating control and treatment group animal runs. Testing started at the same time $(+/-1.5 \mathrm{hr})$ for tests run on more than one day, with early and late times alternated for inter-group randomization.

\section{Open field test}

Testing was carried out in an Open Field apparatus made up of an open topped, clear Plexiglas box, measuring $40.64 \mathrm{~cm} \times 40.64 \mathrm{~cm}$ and $38.1 \mathrm{~cm}$ high. Two rings of photobeams and optical sensors surrounded the box. The optical sensors were connected to a computer by way of an input matrix. Each mouse was placed into the box, and beam interruptions were automatically recorded as a measure of locomotor activity. Each mouse was tested in the box for a period of $30 \mathrm{~min}$.

\section{Barnes maze test}


Barnes Maze (BM) testing was performed a single time only, 14 months post-cell or -control

498

499

500

501

502

503

504

505

506

507

508

509

510

511

512

513

514

515

516

517

518

injection. The BM test is a hippocampus-dependent, spatial-learning task that allows subjects to use spatial cues to locate a means of escape from a mildly aversive environment (i.e. the mice are required to use spatial cues to find an escape location). Mice were assessed for their ability to learn

the location of an escape box over the course of 9 days in the BM apparatus ${ }^{28,29}$. The escape hole is constant for each mouse over the five training days. Each mouse was tested three times per day (3 trials) for 4 days, followed by no testing for 2 days, and re-testing on day 7. A 35-60 min intertrial interval separates each trial. Each trial began by placing one mouse inside a start box with a bottomless cube positioned centrally on the maze. After 30 seconds, the start box was lifted and the mouse was released from the start box to find the one hole with access to the escape box. Two fluorescent lights located approximately 4 feet above illuminated the testing room. Each trial lasted up to 4 min or until the mouse entered the escape box. The experimenter guided mice that failed to find the escape hole within 4 min, to the correct hole after each training test. Once the mouse entered the escape box, it was allowed to remain in the box for $1 \mathrm{~min}$. Following the $7^{\text {th }}$ day of testing, and never on the same day, mice were tested an additional two-days, in which the escape box was placed in the reverse position on day 8, and replaced in the original position on day 9. The same exact testing procedure was applied to all mice in all groups. The maze and all compartments were cleaned thoroughly with isopropyl alcohol to remove any olfactory cues after each trial, and prior to each day of testing. Additional randomization of alternating escape compartment location between each animal per group, and between each of 3 daily training tests per animal, was employed for this test.

\section{Y-maze spontaneous alternation behaviour}


Mice were tested for SA a single time only, at 12 months post-cell or -control injection. Y-

Maze Spontaneous Alternation Behaviour (SAB) is used to assess working memory. SAB was measured by individually placing animals in one arm of a symmetric Y-maze made of opaque black acrylic plastic (arms: $40 \mathrm{~cm}$ long, $4 \mathrm{~cm}$ wide; walls: $30 \mathrm{~cm}$ tall), and the sequence of arm entries and total number of entries recorded over a period of $8 \mathrm{~min}$.

\section{Flinch-jump/fear conditioning tests}

Flinch-jump/Fear Conditioning freezing times were determined 6 and 11 months post-cell or -control injection. We first determined there were no significant differences in the nociceptive threshold (pain sensitivity) across treatment groups using the Flinch-Jump Test. Pavlovian Fear Conditioning was then used to assess learning and memory regarding aversive events. The apparatus (Freeze Monitor ${ }^{\mathrm{TM}}$, San Diego Instruments, San Diego, CA) consisted of a Plexiglas box (25.4 x $25.4 \times 31.75 \mathrm{~cm}$ high) with a stainless-steel grid floor. An acoustic stimulus unit is located on top of the box, and the box is ringed with photo beams and optical sensors. The optical sensors were connected to a computer by way of an input matrix, and the number of beam interruptions is automatically recorded. For testing, on day 1 individual mice were placed into the test box, and allowed to habituate for $3 \mathrm{~min}$. At $3 \mathrm{~min}$ a tone was presented for $30 \mathrm{sec}$. Then, $30 \mathrm{sec}$ after termination of the tone, a $0.5 \mathrm{sec}$ foot shock (intensity = mean jump threshold for the treatment group determined by the Flinch-Jump Test) was delivered. The mouse was then removed from the box and returned to its home cage for $2 \mathrm{~min}$. The chamber was cleaned and the animal returned to the chamber where the procedure was repeated. The freeze monitor apparatus recorded freezing times throughout the procedure (absence of movement for $5+$ seconds, resulting in no beam breaks). On day 2, context retrieval was determined by placing the mouse into the same test box where it previously received a tone and foot shocks, but here the tone and foot shocks were not 
542 presented. Freezing time was measured over a 10-min period. On day 3, cue conditioning was 543 measured after inserting a triangular, plexiglass box into the test box. The mouse was placed into 544 the triangular chamber where they had not previously received tone or foot shocks, but after 1 min 545 the auditory tone was delivered for $30 \mathrm{sec}$ and freezing time measured for $10 \mathrm{~min}$.

\section{Human Subjects}

Cohort 1: 40 control individuals (CTRL; normal aging - normal CSF profile; 16M, 24F; avg age 60.2 yr [49-88]); 52 MCI patients with an AD-characteristic CSF biomarker profile (MCI-AD; 20M, 32F; avg age 75.5 yr [49-88]); 36 MCI patients not displaying an AD-characteristic CSF biomarker profile (MCI; 17M, 19F; avg age 62.22 yr [42-82]); 50 sporadic AD patients with an AD-characteristic CSF biomarker profile (AD; 26M, 24F; avg age 76.08 yr [57-88]). AD biomarker positivity (in-house validated cut-off values of biomarkers were applied: $A \beta_{1-42}<638.5$ $\left.\mathrm{pg} / \mathrm{mL}, \mathrm{T}-\mathrm{tau}>296.5 \mathrm{pg} / \mathrm{mL}, \mathrm{P}-\operatorname{tau}_{(181 \mathrm{P})}>56.5 \mathrm{pg} / \mathrm{mL}\right)$ was determined by means of commercially available single-analyte ELISA kits (INNOTEST® $\beta$-AMYLOID (1-42), INNOTEST® hTAUAg, and INNOTEST® PHOSPHO-TAU (181P); Fujirebio Europe). CSF samples were collected at Middelheim General Hospital (Antwerp, Belgium) and centers referring to the Neurobiobank of the Institute Born-Bunge (NBB-IBB; $n^{\circ}$ BB190113) according to standard collection protocols as described previously ${ }^{30}$. CSF was obtained by lumbar puncture (LP) at the L3/L4 or L4/L5 interspace. CSF samples were collected in polypropylene vials (Nalgene cat.no.5000-1020 $(1.5 \mathrm{~mL})$ and $5000-0050(4.5 \mathrm{~mL}))$, immediately frozen in liquid nitrogen, and subsequently stored at $-80^{\circ} \mathrm{C}$ until analysis.

In addition, blood samples following venepuncture were collected after LP, of which 2-3 serum, 3-4 plasma and 2-3 total blood aliquots were stored (1.5mL for each aliquot). 
564 MCI patients underwent LP at baseline as part of their diagnostic work-up. The inclusion criteria 565 for the control group were: [1] no neurological or psychiatric antecedents and [2] no organic 566 disease involving the central nervous system following extensive clinical examination. MCI 567 patients were diagnosed applying Petersen's diagnostic criteria ${ }^{31}$, i.e., [1] cognitive complaint, 568 preferably corroborated by an informant; [2] objective cognitive impairment, quantified as 569 performance of more than 1.5 SD below the appropriate mean on the neuropsychological subtests; 570 [3] largely normal general cognitive functioning; [4] essentially intact activities of daily living 571 (basic and instrumental activities of daily living were determined by a clinical interview with the 572 patient and an informant); and [5] not demented ${ }^{32}$. AD dementia was clinically diagnosed 573 according to the NINCDS/ADRDA and IWG-2 criteria ${ }^{33}$ APOE allele status was 40\% 3/4, 40\% $5744 / 4,10 \% 3 / 3,10 \% 2 / 4$ for AD group (10 tested/50 total); 55\% 3/4, 20\% 4/4, 15\% 3/3, 10\% 2/4, 575 $0 \%$ 2/3 for MCI-AD group (20 tested/52 total); $31 \%$ 3/4, $0 \% 4 / 4,46 \% 3 / 3,8 \% 2 / 4,15 \% 2 / 3$ for MCI-normal group (13 tested/36 total); 22\% 3/4, 0\% 4/4, 68\% 3/3, 0\% 2/4, 8\% 2/3 for control group (37 tested/40 total). Mean MMSE score was 20 (5-29) +/- 5 for AD group (48/50 tested); 24 +/- 3 (15-30) for MCI-AD group (51/52 tested); 26 +/- 3 (18-30) for MCI-normal group (33/36 tested); $28+/-4$ (17-30) for control group (9/37 tested). All included subjects were of Caucasian 580 ethnicity.

Cohort 2: Cognitive Testing. 29 self-referred memory clinic patients (average age 49; 22-70) from 582 Cedars-Sinai Dept. of Neurosurgery were clinically diagnosed as normal $(\mathrm{n}=6)$, MCI $(\mathrm{n}=18)$, 583 dementia $(n=3)$, or uncertain $(n=2$; diagnosis established solely on basis of cognitive testing), 584 and followed up with cognitive (Montreal Cognitive Assessment; MoCA) testing $(\mathrm{n}=22)$. 585 Cognitively normal $=$ MoCA score 29-30; age-related cognitive decline = MoCA score 26-28; 
MCI $=$ MoCA score $<26$. HLA-A2-negative patients were determined by flow cytometry and excluded from analysis, as were patients with $\leq 2.5 \% \mathrm{CD}^{+}$cells in lymphocyte gates.

Cohort 3: Brain Western and IHC. Hippocampal lysates from 13 autopsy-confirmed Braak stage IV $(n=4)$ and Braak stage V-VI $(n=9)$ sporadic AD patients, and 5 age-matched normal controls were run on Western blots using anti-CD8 or anti-PRF1. Hippocampal sections from 10 autopsyconfirmed Braak stage V-VI sporadic AD patients, and 10 age-matched normal controls were stained with anti-CD8-flourescein plus APP(471-479)/HLA-A2-PE. HLA-A2-negative samples were not excluded from Western and IHC/IF analysis.

\section{Statistical analysis}

Quantification and stereological counting procedure for cell numbers or area $\left(\mu \mathrm{m}^{2}\right)$ of $\mathrm{A} \beta$ plaque, $\mathrm{GFAP}^{+}, \mathrm{Iba}^{+}$or Perforin $1^{+}$cells were analyzed in six to eight coronal sections from each individual, at 150- $\mu \mathrm{m}$ intervals (unless otherwise indicated), covering 900-1200 $\mu \mathrm{m}$ of the hippocampal and cortical areas. Specific fluorescence signal was captured with the same exposure time for each image and optical sections from each field of the specimen were imported into NIH Image $\mathrm{J}$ and analyzed as above. GraphPad Prism (version 5.0b; San Diego, CA, USA) was used to analyze the data using ANOVA and T-Tests with Welch's correction (no assumption of equal variance). In all histograms, average \pm SEM is depicted.

Data from Open Field and Barnes Maze tests were analysed by 2-sided T-Test for individual test points, and by ANOVA on test curves, when normal distribution $/ P>0.05$ of data was verified in Anderson-Darling, D'Agostino \& Pearson, Shapiro-Wilk, and/or Kolmogorov-Smirnov tests. 1sided T-Test was used to analyze Y-maze/SAB. Mann Whitney test was substituted for T-Tests when non-normal distribution $/ P<0.05$ was indicated in Anderson-Darling, D'Agostino \& Pearson, 
Shapiro-Wilk, and/or Kolmogorov-Smirnov tests. Flinch-Jump and Fear Conditioning Tests was normalized, first within each group to the average of the initial two tests in training on day 1 , and then within all experimental groups to the average contextual or cue values of PBS controls, expressed as percent of control, and analyzed by ANOVA, followed where appropriate by Newman-Keuls tests to detect differences among treatment groups.

Sample sizes for PrfKO-CD8 and Ifn $\gamma$ KO-CD8 groups were calculated a priori for each metric using means and standard deviations of PBS and wt-CD8 groups for anticipated effect sizes, with alpha 0.05 , and $>95$ confidence. Calculated $n$ plus $\geq 1$ were then used for PrfKO-CD8 and Ifn $\gamma$ KO-CD8 groups.

Pre-determined exclusions included sections or samples with no discernible background signal, and values within each group $\geq 2$ standard deviations above or below the median/group. Subject numbers and methods of reagent validation are shown in Table S1.

\section{Study approval}

All animal procedures were approved prior to performance by the Cedars-Sinai Institutional Animal Care and Use Committee. The Cedars-Sinai Institutional Review Board designated the analysis of de-identified human brain specimens from UC Davis exempt from committee review.

Brain specimens were collected, stored, and disseminated with prior approval by the UC Davis Medical Center Institutional Review Board. Sampling for cohort 1 was approved by the Medical Ethics Committee of the Hospital Network Antwerp (ZNA), Antwerp, Belgium (approval number 2805 and number 2806). 
630 Results and raw data will be made available upon request. Model Organisms and/or the means to 631 generate them will be made generally available for research (non-commercial) use.

\section{Acknowledgments:}

634 We gratefully acknowledge support in conducting behavioral tests from the Cedars-Sinai Research 635 Institute Biobehavioral Core, and Ms. Hannah Schubloom and Mia Oviatt for excellent 636 administrative support and editing.

\section{Author contributions:}

638 AP performed Western blots, behavioral assays, mouse colony management, and flow cytometry; 639 AR performed, analyzed, and quantified tissue immunostaining, and conducted neuronal counts;

640 MJ performed Western blots, behavioral assays, and ELISAs; RMC provided guidance on model 641 development and analysis, and evaluated the studies' relevance to human conditions; RC 642 performed behavioral assays and mouse colony management; NY performed tissue 643 immunostaining and mouse colony management; RNP designed and analyzed behavioral assays; 644 GD performed Western blots; AM performed Western blots; DG performed flow cytometry; HS 645 performed behavioral assays; L-WJ characterized and provided human brain specimens; DVD, 646 YV, and HDR performed flow cytometry; PPDD characterized and provided human blood 647 specimens, and advised on analysis; KLB evaluated the studies' relevance to human conditions; 648 CJW designed all studies, coordinated experiments, analyzed and compiled all data, and wrote the 649 manuscript. 
651 CW is the author of patents PCT/US2016/049598, WO2017/040594, and PCT/US2019/017879.

$652 \mathrm{RC}$ and KLB are co-authors on patent PCT/US2019/017879. PCT/US2016/049598, WO 653 2017/040594 is licensed by Cedars-SinaiMedical Center to T-Neuro Pharma, Inc. CW has received 654 salary and ownership interest in T-Neuro Pharma, Inc.

\section{Additional information:}

656 Funding: This research was supported in part by UC Davis Alzheimer's Disease Center (P30 657 AG10129), NIH grants to RMC and CJW (R21NSO54162 and R21AG033394, respectively), a 658 grant from the Joseph Drown Foundation (CJW), and the Maxine Dunitz Neurosurgical Institute.

659 Running Title: CD8 T cell aging in Alzheimer's pathology

660 Keywords: Alzheimer's Disease, CD8 T cell, immune aging, homeostatic expansion, 661 neurodegeneration

662 Abbreviations: Alzheimer's disease (AD); Alzheimer's disease transgenic (ADtg); Amyloid 663 Precursor Protein (App); beta-amyloid (A $\beta)$; experimental autoimmune encephalomyelitis (EAE); 664 familial Alzheimer's disease (FAD); Fear Conditioning (FC); hyper-phosphorylated tau protein 665 (pTau); multiple sclerosis (MS); neurofibrillary tangles (NFTs); regions of interest (ROI); 666 Spontaneous Alternation (SA); T Cell Receptor beta (TCRVß); paired-helical filaments (PHFs). 667 Resident memory CD8 T cells (CD8 $\left.\mathrm{T}_{\mathrm{RM}}\right)$.

information:

found

at

https://mtsnature.nature.com/nature_files/2021/02/02/00394605/00/394605_0_data_set_3597679_qnw3pg. 670 docx. 
$671 \quad{ }^{2}$ Correspondence to C.J. Wheeler: chris@ tneuropharma.com

672 
674

675

676

677

678

679

680

681

682

683

684

685

686

687

688

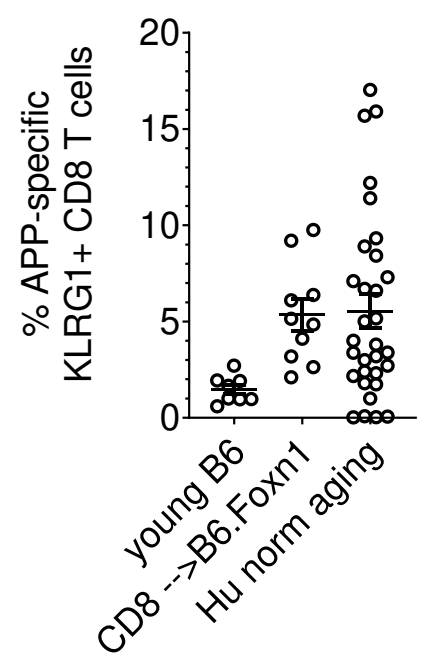

Fig. E1: Flow cytometry and Western analysis of $\mathbf{T}$ cell and amyloid markers. Levels of mouse $\operatorname{APP}_{(470-478)} / \mathrm{H}-2 \mathrm{D}^{\mathrm{b}}$ and human $\mathrm{APP}_{(471-479)} / \mathrm{HLA}-\mathrm{A} 2$ were quantified within $\mathrm{KLRG}^{+} \mathrm{CD} 8 \mathrm{~T}$ cells by flow cytometry in mouse and human blood, respectively. Young B6 = APP-reactive T cells within splenic lymphocytes from 8-10 week-old C57BL/6 females; CD8 $\rightarrow$ B6.Foxn1 = APP-reactive T cells within splenic lymphocytes from 10-12 week-old B6.Foxn1 female recipients of purified CD8 T cells from 6-8 week-old C57BL/6 females 3-5 weeks prior; Hu norm aging = APP-reactive T cells within peripheral blood lymphocytes of neurodegenerative disease-free human subjects aged 49-88 years, whose values also appear in Fig. 4c. 


\begin{tabular}{|c|c|c|c|c|c|}
\hline 50 & $\underline{3 w k}$ & $10 \mathrm{wk}$ & $\underline{6 \mathrm{mos}}$ & $11-15 \mathrm{mos}$ & $15 \mathrm{mo}$ \\
\hline$\frac{\text { Analysis }}{\text { \& Endpts: }}$ & 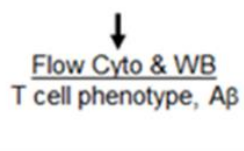 & 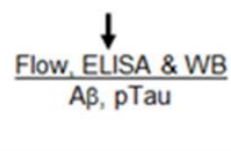 & $\frac{\begin{array}{c}\downarrow \\
\text { Fear Cond, OFT }\end{array}}{A \beta \text { IF }}$ & $\frac{\begin{array}{l}\downarrow \\
\text { Behavior }\end{array}}{\mathrm{A} \beta \mathrm{IF}}$ & 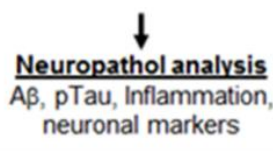 \\
\hline
\end{tabular}

691

692

693

694

695

696

697

698

699

700

701
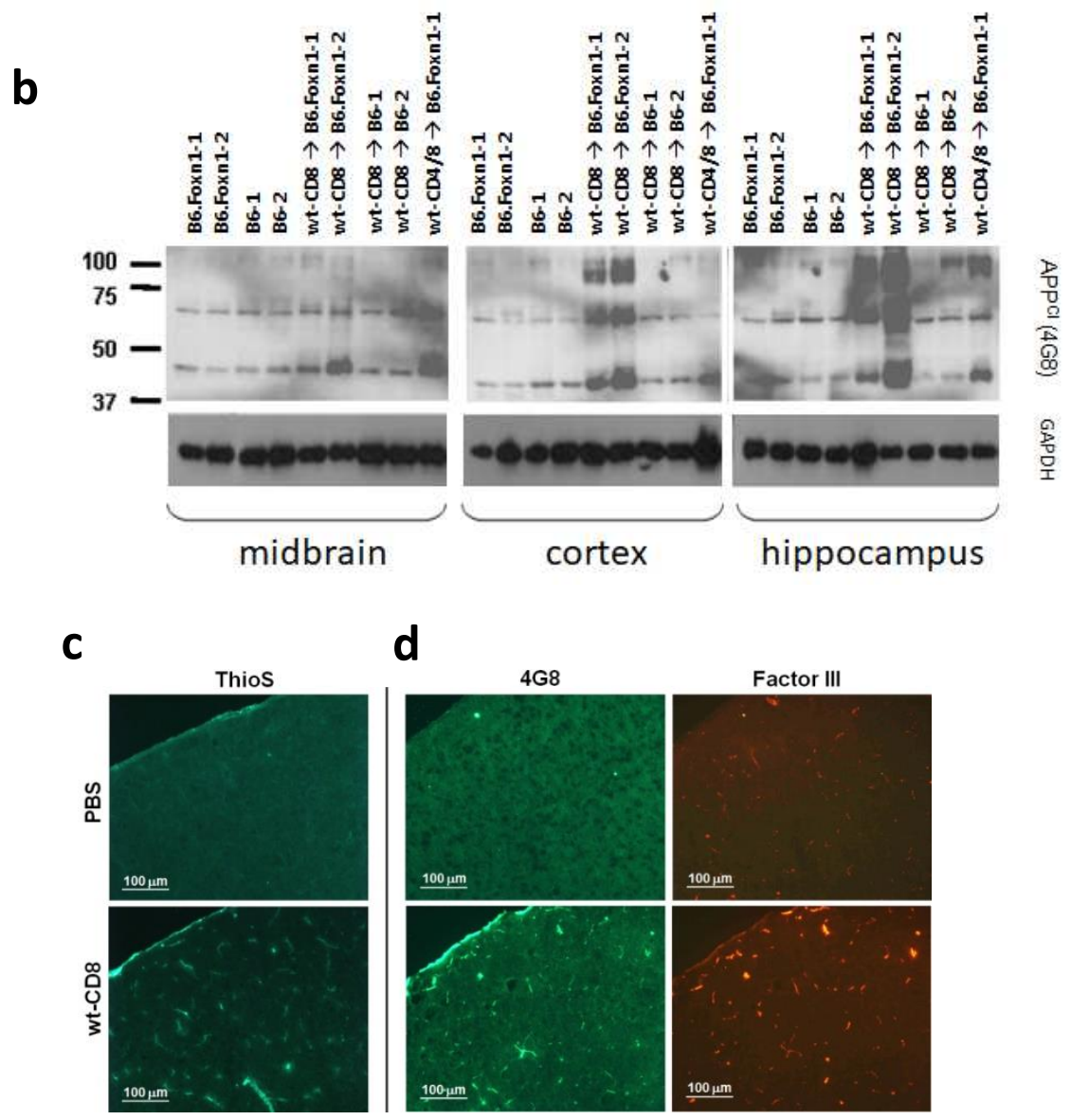

Fig. E2: Analysis scheme, APP cleavage products, and vascular A $\beta$ in brain after $T$ cell injection. Analysis time line in CD8 T cell-injected B6.Foxn1 recipients (a). Western analysis of APP cleavage products/A $\beta$ oligomers $\left(\mathrm{APP}^{\mathrm{Cl}}\right)$ with $4 \mathrm{G} 8$ antibody in dissected regions of mouse brain 10 weeks after injection with $3 \times 10^{6}$ purified T cells. CD4/8 indicates injection of $\sim 85 \%$ CD4 and 15\% CD8, with peripheral proportions verified by flow cytometry (b). 
702 Immunohistochemical staining for ThioS in B6.Foxn1 cortex 6 months post-injection, exhibiting 703 vascular staining pattern in wt-CD8 T cell-injected, but not PBS-injected mice (c). A $\beta$ (4G8) and 704 Factor III staining in B6.Foxn1 cortex 6 months after injection, confirms deposits of aggregated 705 vascular $\mathrm{A} \beta$ in nude mice harboring hi $\mathrm{T}$ cells $(\mathbf{d})$. All images at 5x magnification. 
a

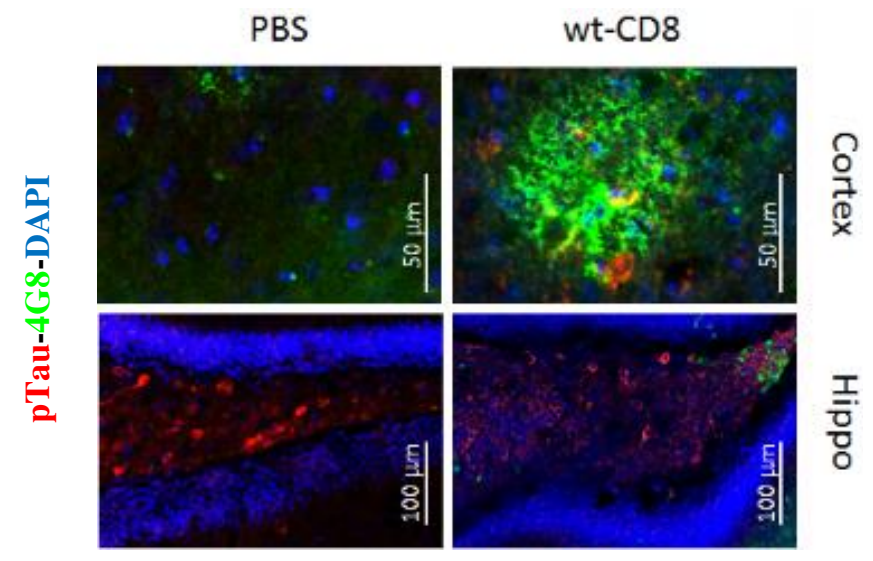

b

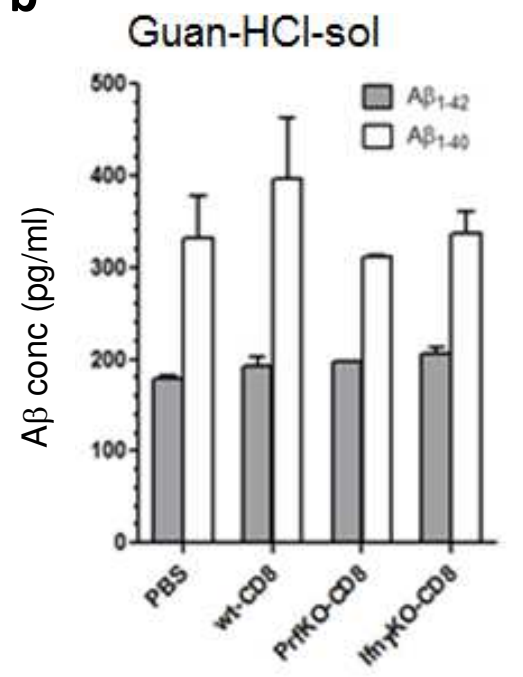

725

726 Fig. E3: $\mathbf{A} \boldsymbol{\beta}$ accumulation in nude mouse brain after $\mathbf{C D 8} \mathbf{T}$ cell injection. Representative example of individual $\mathrm{A} \beta\left(4 \mathrm{G}^{+}\right)$plaque morphology within entorhinal cortex and hippocampus

728 in nude recipients 15 months after CD8 $\mathrm{T}$ cell injection. Magnification and image acquisition

729 parameters were identical within each brain region (a). Forebrain ELISA of GuanidineHCl730 soluble $\mathrm{A} \beta$ in B6.Foxn1 brain 15 months after CD8 $\mathrm{T}$ cell or control injections (b). 

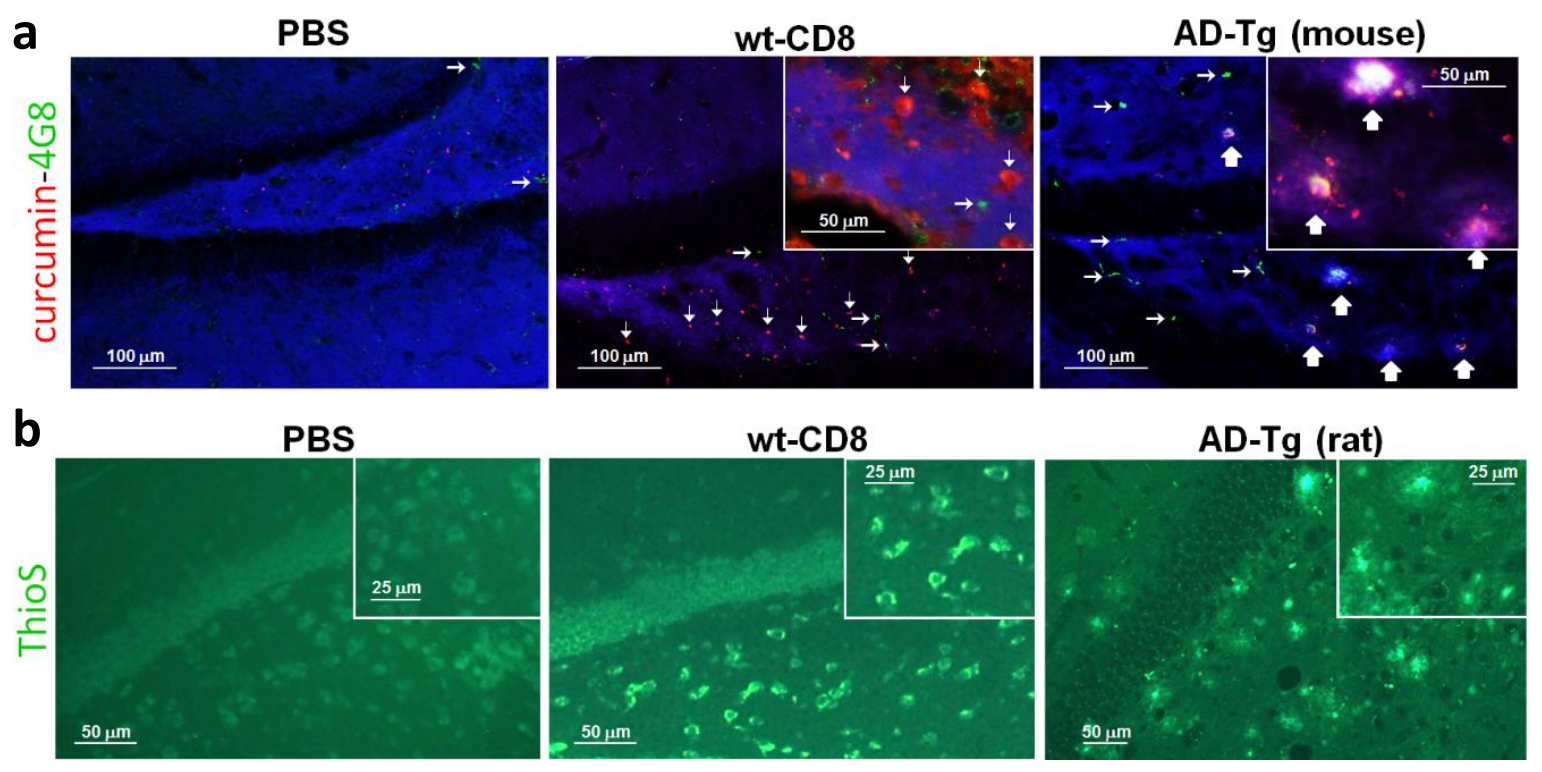

Fig. E4: Distinct curcumin and ThioS staining in dentate gyrus of nude mice harboring hiT

cells. a, Hippocampal sections from the indicated groups (all B6.Foxn1 recipients, except AD-Tg $=\mathrm{Tg} 2576$ mice), were stained for 4G8 (A $\beta)$ and curcumin, 6 months after i.v. control/cell injection, or at 14 months of age for AD-Tg (a). Right-facing arrows highlight $\mathrm{A} \beta$ deposits with no curcumin co-staining. Up-facing arrows depict co-localized $A \beta$ and curcumin deposits, representing mature neuritic plaques. Down-facing arrows highlight curcumin ${ }^{+}$structures with no A $\beta$ co-staining, i.e., non-amyloid fibrillar deposits. No DAPI was used in the stains; blue channel background is provided for anatomical context only. Follow-up ThioS staining of PBS and wt-CD8 group B6.Foxn1 hiT recipients 6 months after control/cell injection, and 20 month-old AD-Transgenic et al., 2013), that nevertheless failed to stain with ThioS in our hands. 

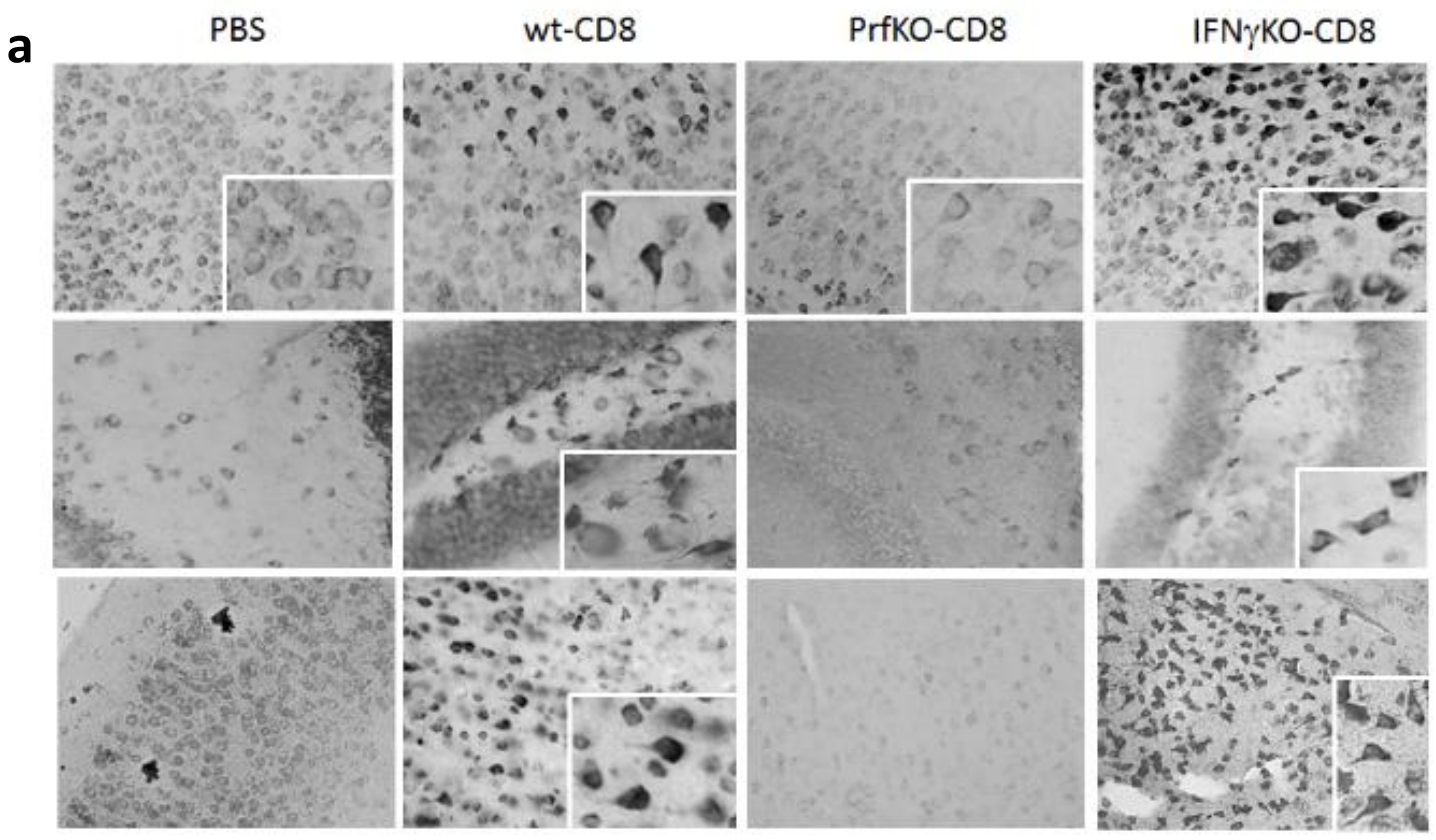

퐇

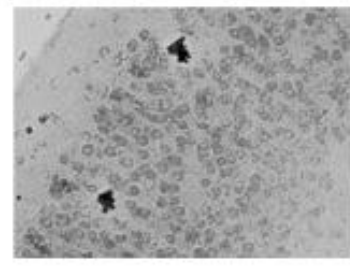

b
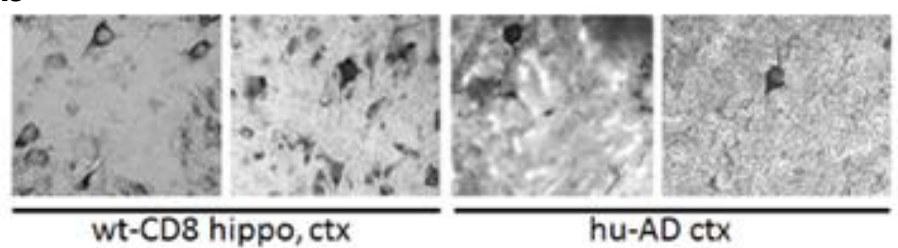

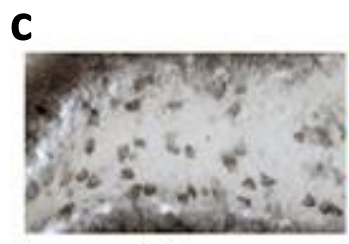

Galyas

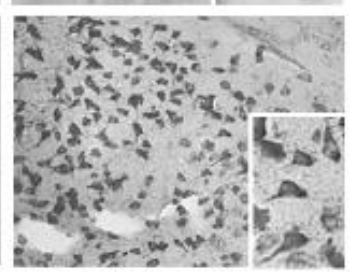

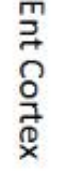

751

752

753

754

Fig. E5: Silver stained neuronal structures in experimental groups. Gallyas silver staining of

$$
\text { cortical and hippocampal brain regions, showing typical neurofibrillary tangle (NFT) morphology }
$$

in wt-CD8 and IFN $\gamma \mathrm{KO}-\mathrm{CD} 8$ group mice (insets). Background silver staining was occasionally evident in PrfKO-CD8 or PBS group mice, but did not exhibit similar NFT morphology (insets). Individual images were derived from different mice within each group (a). Comparison of Gallyas ${ }^{+}$ structures in nude mice harboring ${ }^{\text {hi }} \mathrm{T}$ cells (wt-CD8) hippocampus (left) and cortex (ctx, right), to those in cortex of human severe AD (Braak stage VI; b). Sequential staining of wt-CD8 group mouse hippocampus reveals identical staining pattern between Gallyas and pTau, but not Gallyas and 4G8/A $\beta$ (c). Magnification and scale are identical for all images (20x), and among insets in $\mathbf{a}$. 

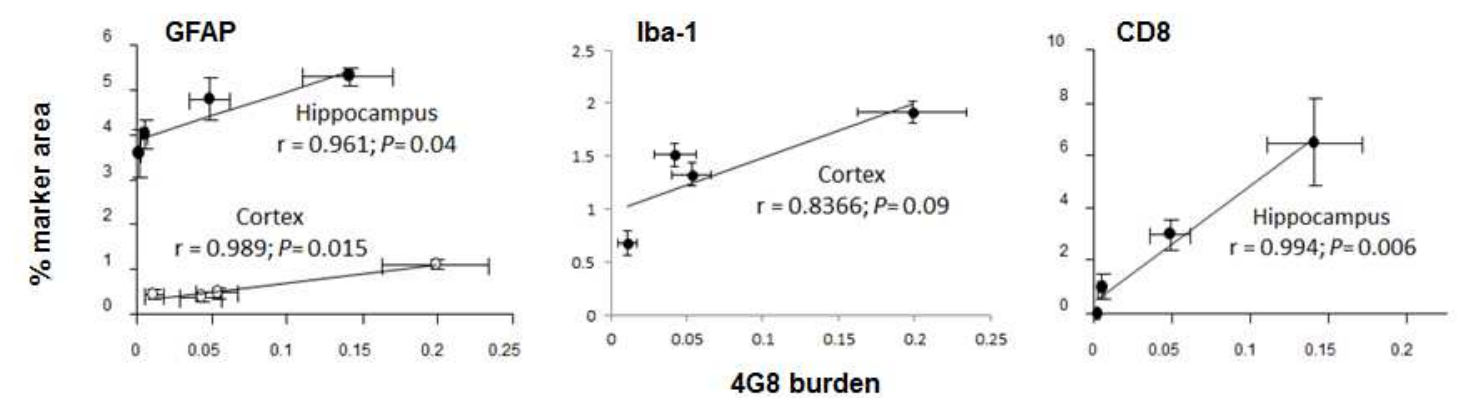

Fig. E6: Innate and adaptive immune correlates of amyloidosis in nude mice harboring hiT cells. Brain was co-stained for CD8 and pTau (inset), and quantified within hippocampal and cortical brain sections from B6.Foxn1 recipients 15 months after injection of wild-type, IFN $\gamma \mathrm{KO}$ or PrfKO CD8 T cells, or PBS as previously reported (Panwar et al., 2020; reference 7 in manuscript). Group data are compiled for Astrocytic (GFAP), microglial (Iba-1), and CD8 T cell (CD8) areas were correlated with $4 \mathrm{G}^{+}$plaque burden within each group, with $P$ values of linear regressions and Pearson's correlations (r) shown $(\mathbf{C})$. Numbers of mice per group are listed in Supplemental Table 1. 
a

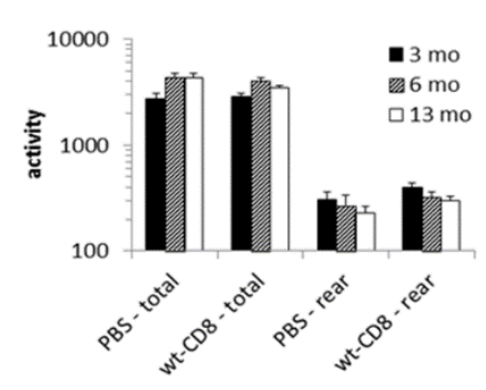

d

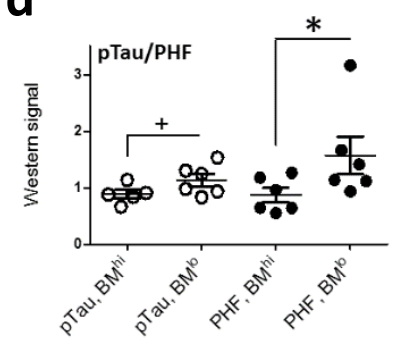

b

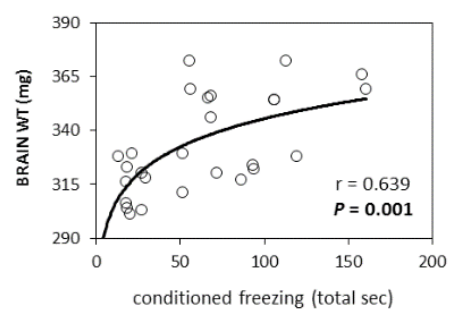

e

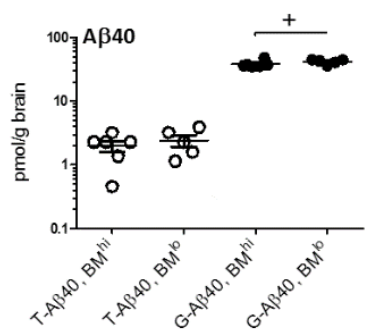

C

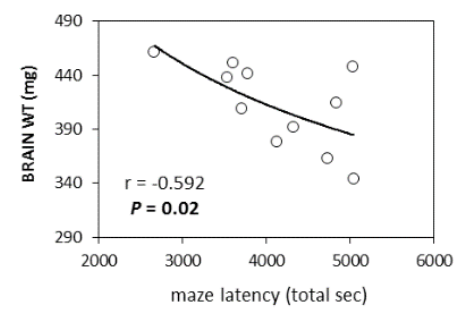

f

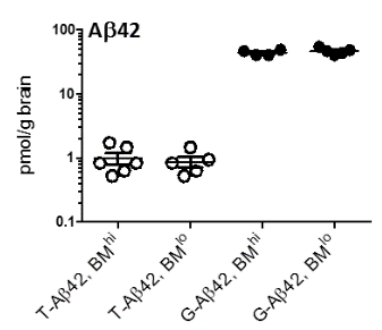

Fig E7: Motor activity, cognitive performance and correlation with pathological features.

Open Field total activity and rearing activity at 3, 6, and 13 months post-injection of CD8 T cells in experimental mouse groups (a). There was no substantial alteration in total or rearing activity between PBS and wt-CD8 groups at any time point, although total activity significantly increased after 3 months, and rearing activity significantly decreased by 13 months, in both groups ( $\mathrm{n} \geq 9$ mice/group). Individual mouse performance in Fear Conditioning test at 6 months correlated directly with brain mass $(n=27$; mice were from PBS and wt-CD8 groups; b). Superior performance of individual mice in Barnes Maze at 14 months was significantly associated with higher brain mass $\left(\mathrm{n}=9\right.$; mice were from all groups; $* P>0.05,{ }^{+} P>0.1$ by 2 -tailed T-test; $\left.\mathbf{c}\right)$. Stratification by median latency in Barnes Maze correlated significantly with Tau PHF only (d), although marginal non-significant trends were observed for pTau and GuanidineHCl-soluble Ab40 relative to Triton $\mathrm{X}-100$-soluble $\mathrm{A} \beta$ species $\left(\mathrm{G}-\mathrm{A} \beta\right.$ and $\mathrm{T}-\mathrm{A} \beta$, respectively; $\mathrm{BM}^{\mathrm{hi}}=$ longer latency; $\mathrm{BM}^{\mathrm{lo}}=$ shorter latency; $\left.\mathbf{e}, \mathbf{f}\right)$. 

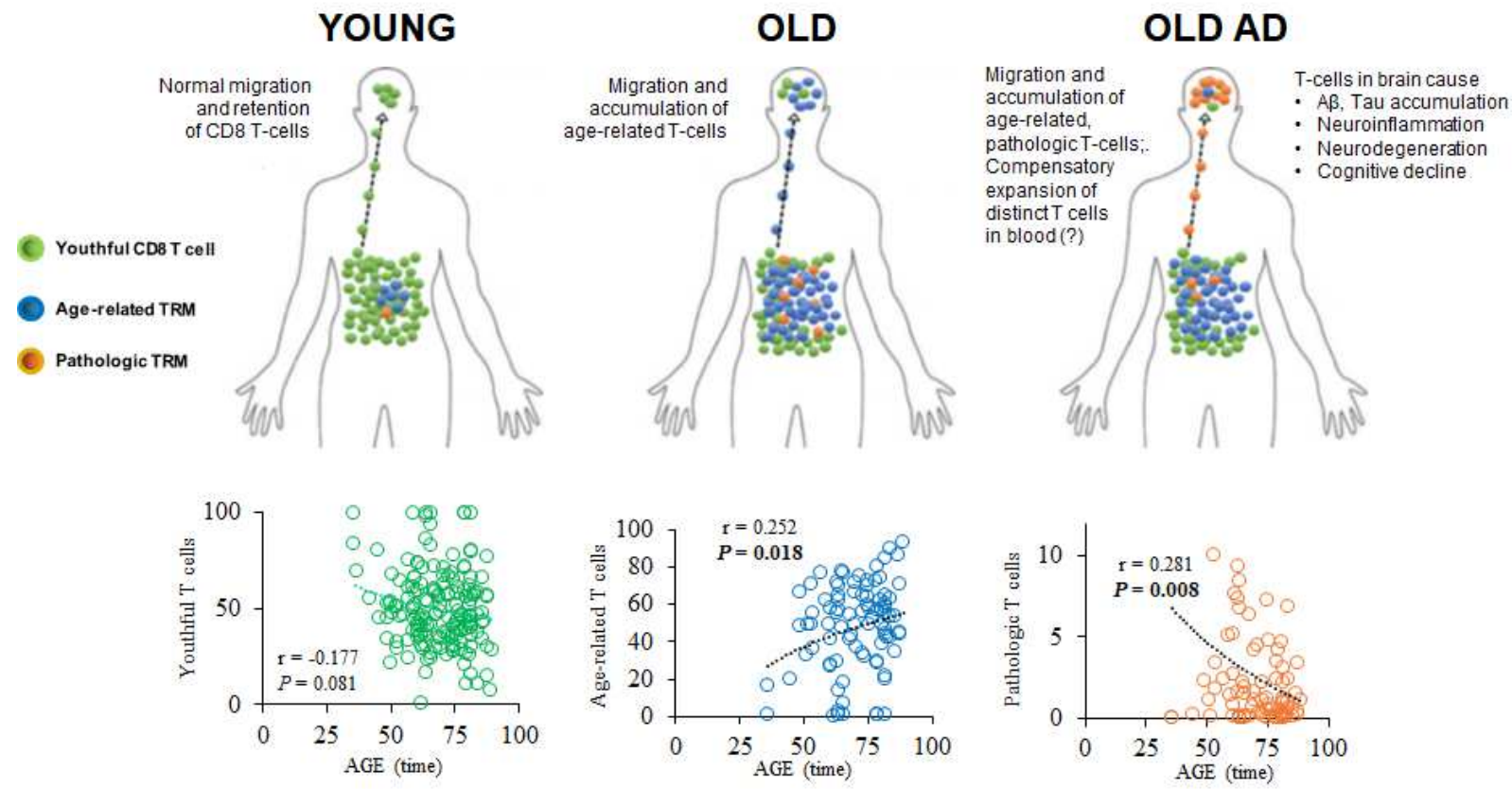

794
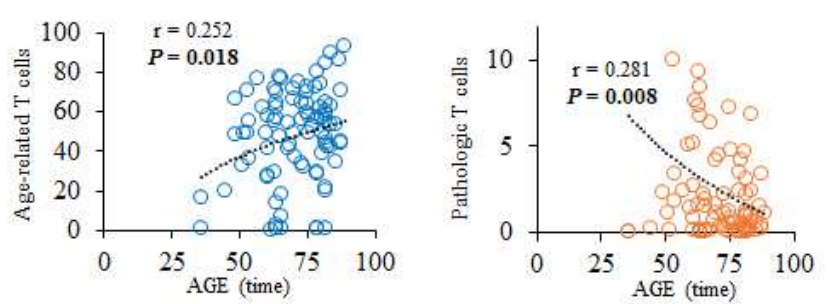

795

796

Fig. E8: KLRG1" ("youthful"), KLRG1+ ("age-related"), and APP(471-479)/HLA-A2 multimerreactive KLRG1+ ("pathologic") CD8 T cells exhibit differential associations with age. Whole blood from 165 human patients (31 MCI - norm bio; 45 MCI - AD bio; 40 normal aging controls; 49 AD) was analysed by flow cytometry for $\mathrm{KLRG}^{+}$and KLRG1- CD8 $\mathrm{T}$ cell content in lymphocyte gates, and plotted relative to patient age at blood collection, with $\mathrm{APP}(471-479) / \mathrm{HLA}$ A2 multimer reactivity within $\mathrm{KLRG}^{+} \mathrm{CD} 8 \mathrm{~T}$ cells further quantified in the subset of 88 HLA803 $\mathrm{A} 2^{+}$patients. Possible migration of age-related and APP-reactive $\mathrm{T}$ cell subsets from blood to brain as depicted above the plots is proposed. 

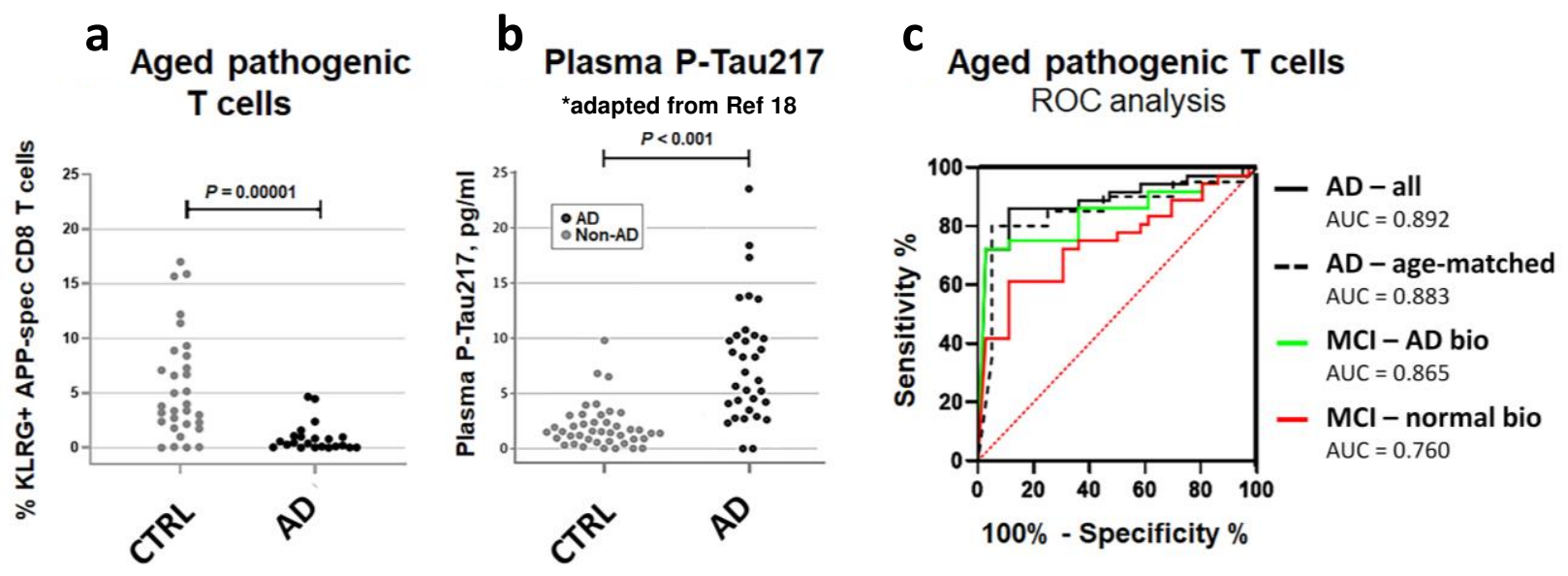

808

809

810

811

812

813

814

815

816

817

818

819

820

821

Fig. E9: Comparison with P-Tau217 and receiving operator characteristic (ROC) curves for APP-specific KLRG1+ CD8 T cell level in human patients. Levels of APP/HLA-A2 multimerreactive $\mathrm{KLRG}^{+} \mathrm{CD} 8 \mathrm{~T}$ cells in blood of $\mathrm{AD}$ patients from our study (a) relative to published plasma levels of P-Tau217 (Palmqvist et al., 2020. JAMA 324(8):772-781; b). Receiver Operating Characteristic (ROC) plots of APP/HLA-A2 multimer-reactive KLRG1 ${ }^{+}$CD8 T cells in blood of indicated patient cohorts relative to normal aging controls (c) Mild Cognitive Impairment without (MCI-normal bio) and with (MCI-AD bio) CSF biomarkers consistent with AD, and confirmed AD patients ages 57-84 (AD-all). Area Under the Curve (AUC) is indicated. AD-age-matched indicates ROC analysis on $10 \mathrm{AD}$ patients for whom precisely age-matched controls were available $(+/-1$ year; $\mathrm{n}=10) . P<0.001$ for all curves except $\mathrm{MCI}-$ normal bio $(P=0.003)$. 


\section{Figures}
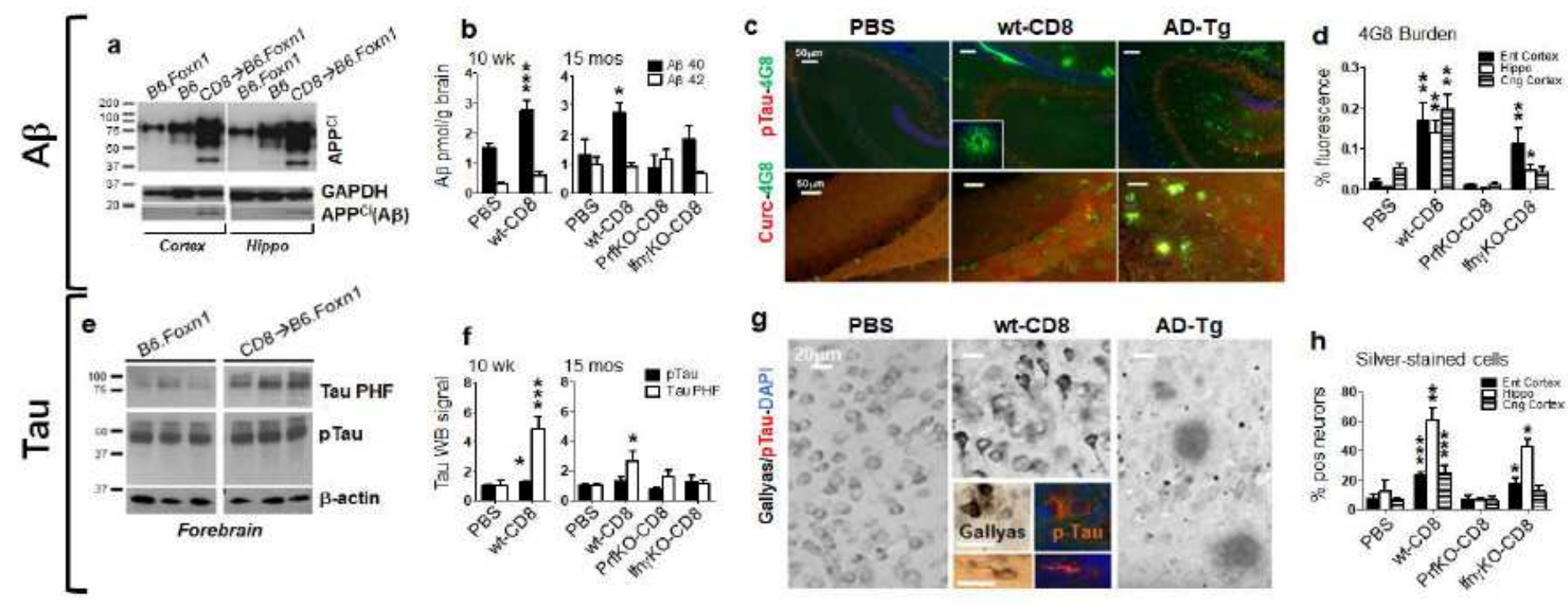

Figure 1

Amyliod and pTau pathology. Western blots of APP cleavage products (APPCl) in cortex, hippocampus 3 wk after injection $(\mathbb{~})$ of recipients (a). B-J depict B6.Foxn1 recipients, 15 mos post-injection unless otherwise indicated. Forebrain A 1 1-40/42 ELISA (b). Plaques + pTau/curcumin staining (c), and compiled 4G8 burden in entorhinal (Ent)/cingulate (Cng) cortex, and hippocampus (Hippo) (d). Western blots (e), and compiled pTau and PHF signal. Gallyas/silver-stained cells in hiTRM groups and 18-month-old Tg2576 (AD-Tg) mice, with sequential pTau囚Gallyas stains inset (g). Gallyas+ neuron (h) percentages Plots depict averages $+\mathrm{SEM} .{ }^{*} \mathrm{P}<0.05,{ }^{\star \star} \mathrm{P}<0.01,{ }^{\star \star *} \mathrm{P}<0.005$ by 2 -sided T-test, relative to PBS group. 

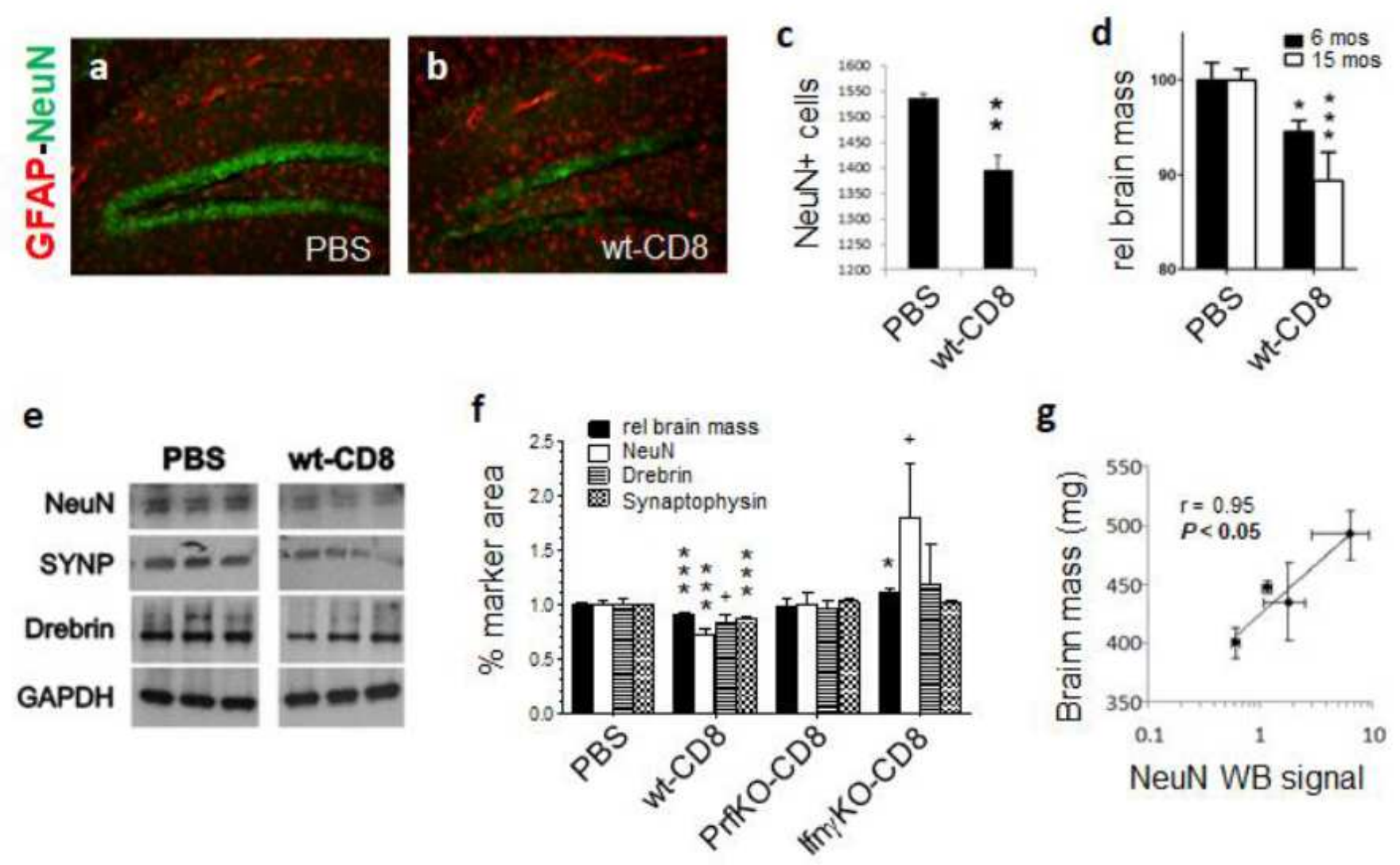

Figure 2

Neurodegeneration in nude mice harboring hiT cells. Cell/control recipients in all panels are B6.Foxn1 exclusively. NeuN and GFAP staining ( $a, b)$, and cell counts in hippocampus, 15 mos after cell/control injection (c). Brain atrophy over time in PBS and wt-CD8 groups (mass normalized to PBS controls at each time point; d). Representative forebrain Westerns (e), and GAPDH-normalized NeuN, Drebrin, and Synaptophysin Western signals (f). Correlation of NeuN with brain weight (g). Plots depict averages + SEM. ${ }^{*} \mathrm{P}<0.05,{ }^{*} \mathrm{P}<0.01,{ }^{* \star *} \mathrm{P}<0.005$ by 2 - sided T-test, relative to $\mathrm{PBS}$ group. 

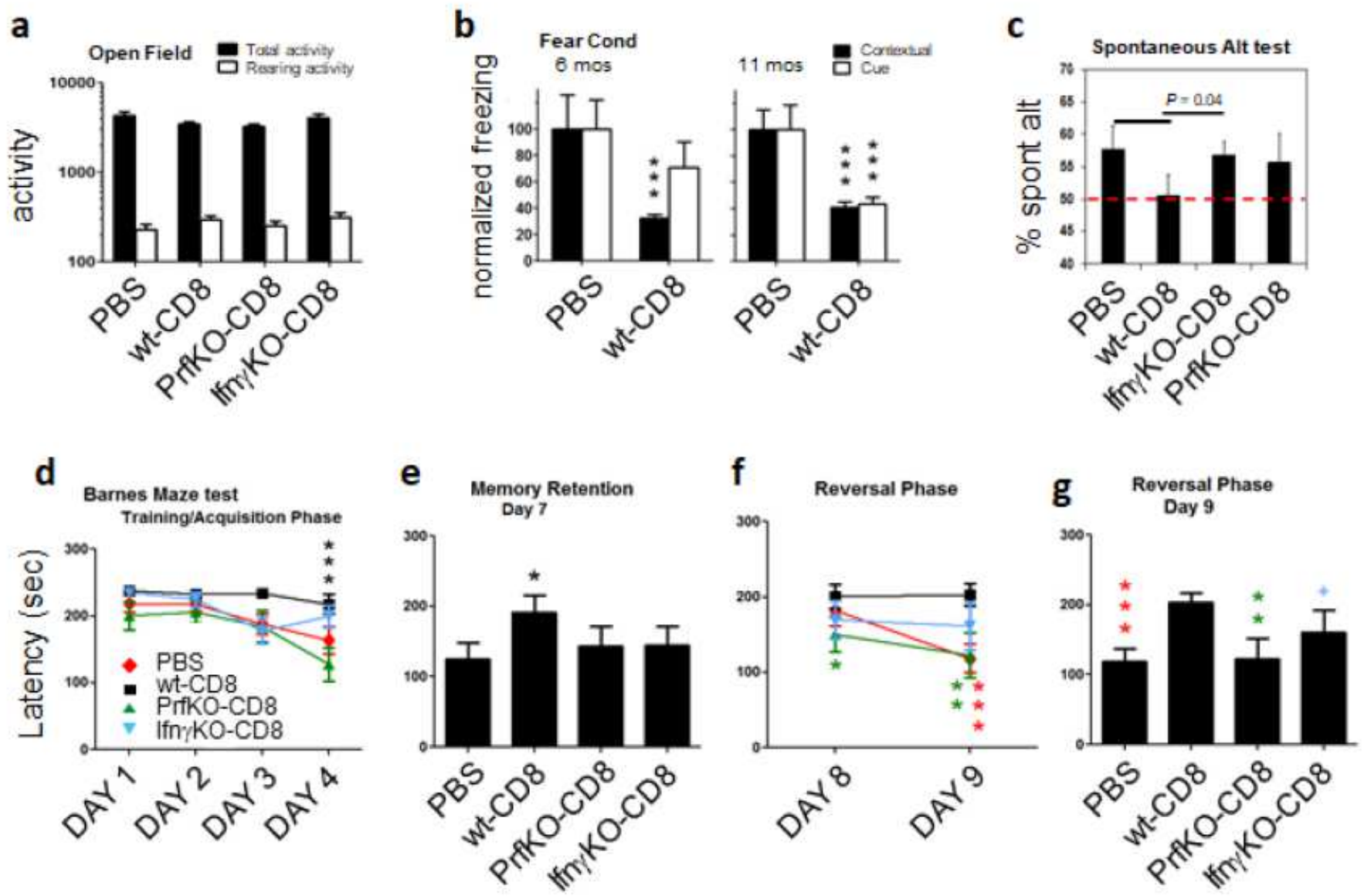

Figure 3

Cognition in nude mice harboring hiT cells. Representative Open Field test at 13 mos (a). Fear Conditioning over time (b), and Spontaneous Alternation Behavior at 12 months (c). Barnes Maze learning/training $(\mathrm{d})$, retention $(\mathrm{e})$, and reversal $(\mathrm{f}, \mathrm{g})$ phases, at 14 mos (black, colored symbols $=\mathrm{P}$ relative to PBS, wt-CD8, respectively). Plots depict averages + SEM. ${ }^{*} P<0.05,{ }^{*} \mathrm{P}<0.01$, ${ }^{\star \star *} \mathrm{P}<0.005$ by 2-sided ANOVA (panel d) or 2-sided T-test (all others), relative to PBS group unless otherwise indicated. 
a

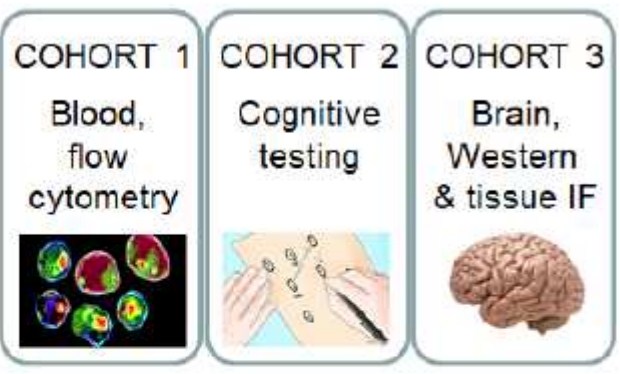

d

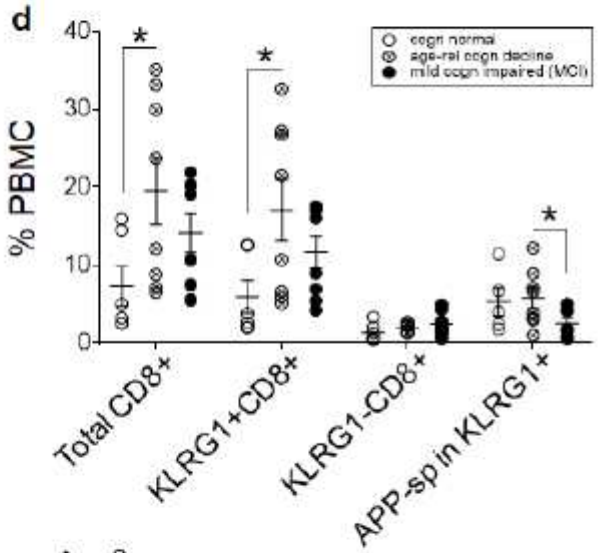

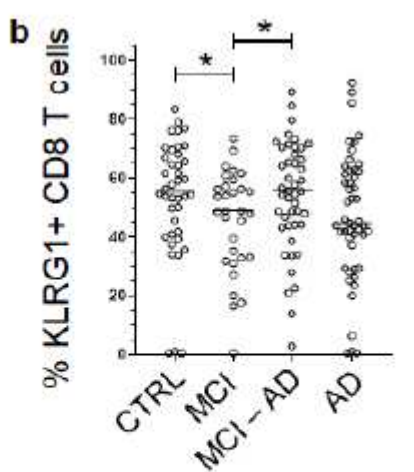

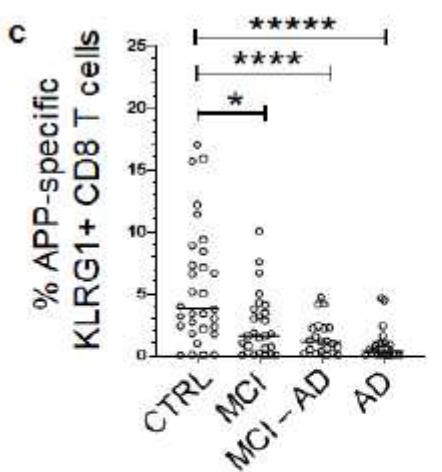

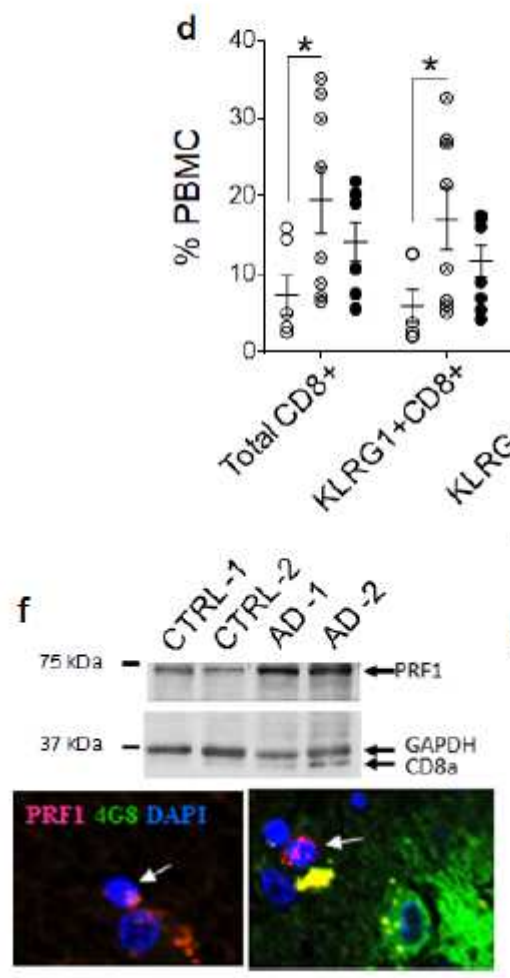
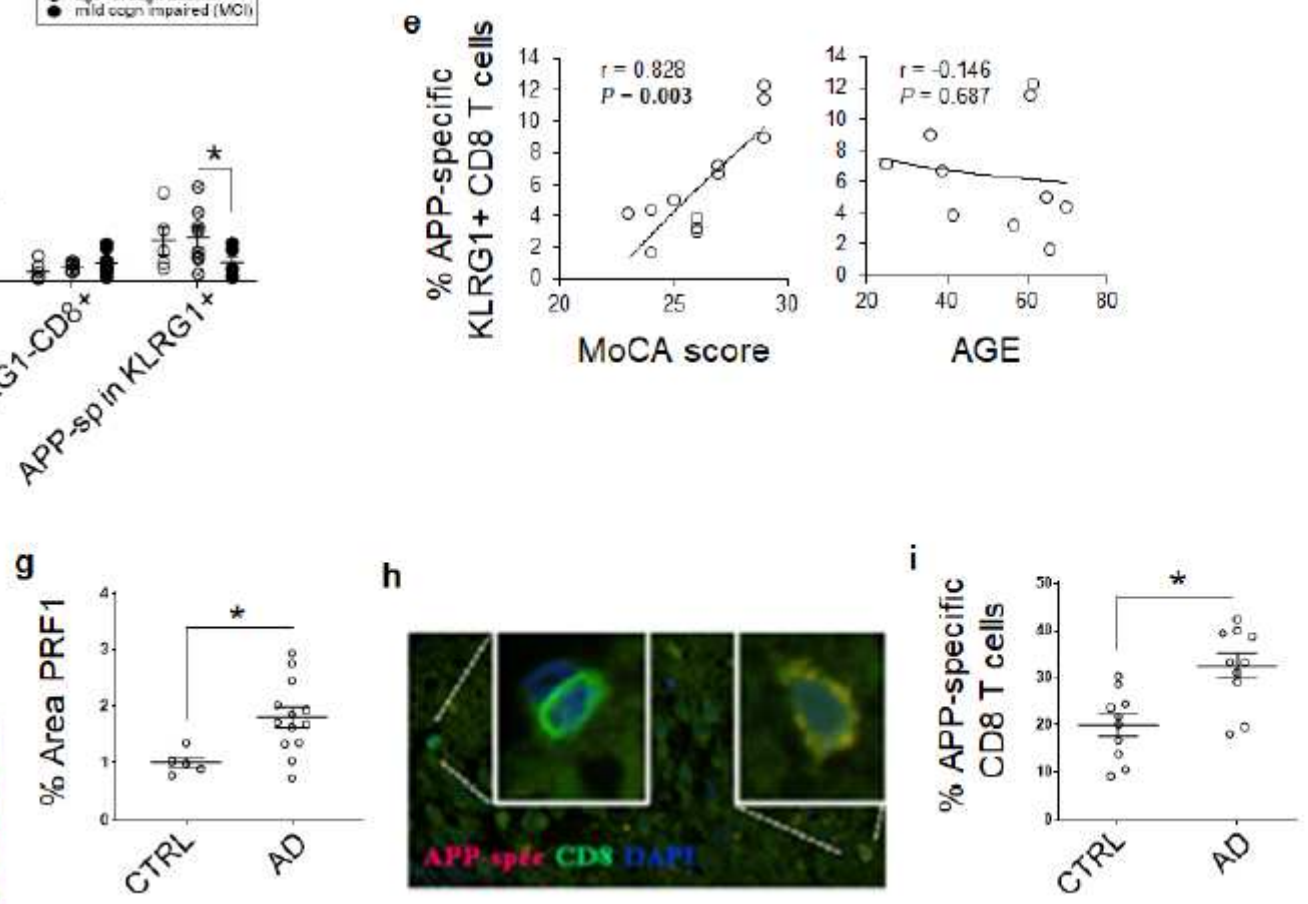

Figure 4

hiT parameters in human Alzheimer's. Patient cohorts (a). KLRG1+ (b) and APP(471- 479)/HLA-A2-

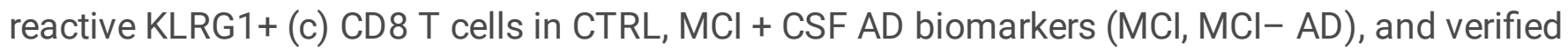
Alzheimer's (AD) blood. T cell subpopulations vs. MoCA score (d), and correlation of APP(471-479)/HLAA2-reactive KLRG1+ CD8 with score and age (e). PRF1 Western blot and immunofluorescence (f), with quantifications in age-matched CTRL and AD brains. (g). APP(471-479)/HLA-A2-reactive CD8 staining (h) and quantification (i) in brain. Plots depict averages + SEM. ${ }^{\star} P<0.05, * \star P<0.01, * \star * P<0.005, * \star \star \star P<$ 0.001 by 2 -sided T-test, relative to CTRL unless otherwise indicated.

\section{Supplementary Files}

This is a list of supplementary files associated with this preprint. Click to download.

- NatureSupplementallnformationclean12520b.docx 